\title{
A Novel Intelligent System Based on Adjustable Classifier Models for Diagnosing Heart Sounds
}

\section{Shuping Sun ( $\nabla$ Shuping.Sun@lEEE.org )}

Tingting Huang

Nanyang Institute of Technology

\section{Biqiang Zhang}

Nanyang Institute of Technology

\section{Peiguang He}

Nanyang Institute of Technology

\section{Long Yan}

Shenzhen University

\section{Dongdong Fan}

Nanyang Institute of Technology

Jiale Zhang

Nanyang Institute of Technology

Jinbo Chen

Nanyang Institute of Technology

\section{Research}

Keywords: Heart sound, Short time modified Transform (STMHT), PCA, GMM, Mahalanobis Distance, Chisquared distribution $(\chi 2)$

Posted Date: February 17th, 2021

DOI: https://doi.org/10.21203/rs.3.rs-213248/v1

License: (c) (1) This work is licensed under a Creative Commons Attribution 4.0 International License. Read Full License 


\title{
A Novel Intelligent System Based on Adjustable Classifier Models for Diagnosing Heart Sounds
}

\author{
Shuping Sun ${ }^{1}$, Tingting Huang ${ }^{1}$, Biqiang Zhang ${ }^{1}$, Peiguang $\mathrm{He}^{1}$, Long Yan $^{2}$, Dongdong Fan ${ }^{1}$ \\ , Jiale Zhang ${ }^{1}$ and Jinbo Chen ${ }^{1}$
}

\footnotetext{
*Correspondence:

Shuping.Sun@IEEE.org

${ }^{1}$ Department of Information

Engineering, Nanyang Institute of

Technology, Nanyang, China

Full list of author information is

available at the end of the article
}

\begin{abstract}
Background: As an efficient method, heart sounds (HSs) analysis by classifying the features extracted from the four-stage sequence consisting of the first heart sound $\left(S_{1}\right)$, second heart sound $\left(S_{2}\right)$, duration from $S_{1}$ to $S_{2}$ and duration from $S_{2}$ to $S_{1}$, has been widely used to diagnose heart disease and evaluate heart functions. However, the feature is difficult to be extracted with high accuracy due to the four stages segmented from HSs with low accuracy; the fixed classifiers achieved by training the features from the old samples cannot better fit the features from the new ones because they are not adjusted with the incremental features. Thus, a novel intelligent diagnostic system, the innovations of which are primarily reflected in the automatic feature extraction and adjustable classifier models, is proposed to realize the diagnosis of heart diseases with higher accuracy.
\end{abstract}

Methods: The three stages of the proposed system are summarized as follows. In stage 1, the short time modified Hilbert transform (STMHT)-based curve is used to segment and extract the first complex sound $\left(C S_{1}\right)$ and second complex sound $\left(C S_{2}\right)$. In stage 2, the envelopes $C S_{1 \mathrm{~F}_{\mathrm{E}}}$ and $C S_{2 \mathrm{~F}_{\mathrm{E}}}$ for periods $C S_{1}$ and $C S_{2}$ are obtained via a novel method, and the frequency features are automatically extracted from $C S_{1 \mathrm{~F}_{\mathrm{E}}}$ and $C S_{2 \mathrm{~F}_{\mathrm{E}}}$ by setting different threshold value (Thv) lines. Finally, the principal component analysis (PCA)-based first three principal components $\gamma_{1}, \gamma_{2}$, and $\gamma_{3}$ are determined as the diagnostic features. In stage 3, a Gaussian mixture model (GMM)-based objective function $f_{\text {et }}(\mathbf{x})$ is generated. Then, the $\chi^{2}$ distribution for component $k$ is determined by calculating the Mahalanobis distance from $\mathbf{x}$ to the class mean $\mu_{k}$ for component $k$, and the confidence region of component $k$ is determined by adjusting the optimal confidence level $\beta_{k}$ and used as the criterion to diagnose HSs.

Results: The performance evaluation was validated by sounds from online $\mathbf{H S}$ databases and clinical heart databases. The accuracy of the proposed method was compared to the accuracies of other well-known classifiers, and the highest classification accuracies of $99.43 \%, 98.93 \%, 99.13 \%, 99.85 \%, 98.62 \%, 99.67 \%$ and $99.91 \%$ in the detection of MR, MS, ASD, NM, AS, AR and VSD sounds were achieved by setting $\beta_{k}(k=1,2, \ldots, 7)$ to $0.87,0.65,0.67,0.65,0.67,0.79$ and 0.87 , respectively.

Conclusions: This proposed intelligent diagnosis system provides an efficient way to diagnose seven types of heart diseases. In addition, methods to manage the sounds (such as some ASD sounds) when $C S_{1}$ and $C S_{2}$ cannot be segmented and extracted via the STMHT method will be explored in the future to characterize the physical meanings of the frequency components and to build a model of the secondary curve in the frequency domain.

Keywords: Heart sound; Short time modified Transform (STMHT); PCA; GMM; Mahalanobis Distance; Chi-squared distribution $\left(\chi^{2}\right)$. 


\section{Background}

As an efficient method, using heart sound (HS) analysis is often used to evaluate heart function; this approach has been widely used to diagnose heart disease and evaluate heart functions, such as congenital heart disease classification [1], ventricular septal defect detection [2], blood pressure estimation [3] and congenital heart disease screening [4], for children and adults. A normal HS is primarily composed of two basic sounds: the first sound $\left(S_{1}\right)$ which is generated by the closing of aortic valves and the vibrations associated with tensing of the chordae tendineae and the ventricular walls, the second sound $\left(S_{2}\right)$ is produced by the closure of the aortic and pulmonic valves at the beginning of is volumetric ventricular relaxation. However, HSs with unitary murmurs generally occur between $S_{1}$ and $S_{2}$ with different noise patterns [5]. Therefore, analyses of $S_{1}, S 2$, and the period between $S_{1}$ and $S_{2}$ play important roles in characterizing $\mathbf{H S}$ features with different types of information. Detailed information for $S_{1}, S_{2}$, and the sounds between $S_{1}$ and $S_{2}$ can be used to accurately classify HS. Additionally, to avoid analyzing the sounds between $S_{1}$ and $S_{2}$, which are generally segmented from HSs with low accuracy, $S_{1}$ and part of the period between $S_{1}$ and $S_{2}$ are integrated to obtain $C S_{1}$, and $S_{2}$ and the part of the period between $S_{1}$ and $S_{2}$ are integrated to form $C S_{2}$. Then, the features are efficiently extracted from $C S_{1}$ and $C S_{2}$. Finally, a classification method is established to diagnose heart diseases.

The studies regarding HS segmentation can be summarized into two branches: one branch includes studies that segment each cardiac cycle into a sequence of four heart stages: $S_{1} \longrightarrow$ Systole period $\longrightarrow S_{2} \longrightarrow$ Diastole period [6, 7]. As a result, the four fundamental stages to be segmented are different due to the nonstationary nature of an abnormal HSs signal and the effect of background noise. The other branch includes studies that segment a periodic HSs into a sequence of two heart stages, which are expressed as $\overbrace{\text { One part of Diastole } \rightarrow S_{1} \rightarrow \text { One part of Systole }}^{C S_{1}} \rightarrow$

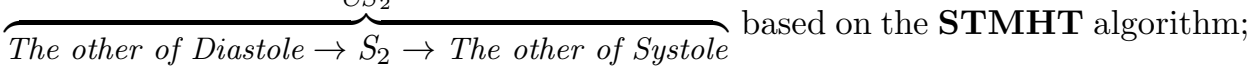
this approach was reported to be successfully applied in diagnosing heart diseases, such as in ventricular septal defect (VSD) diagnosis [8] and several kinds of heart disease diagnosis [9]. Moreover, study [9] noted that the use of frequency features was more efficient in distinguishing normal from abnormal sounds than was the use of time features. Therefore, an efficient frequency feature extraction method should be developed.

As an important component of efficient feature extraction, the frequency width of the envelope over a given threshold value ( $T h v)$ has been verified to be useful for detecting heart diseases $[8,9,10,11]$. However, for many types of HSs, it is difficult to extract frequency widths with an unsuitable Thv due to the existence of a nonsmooth envelope. To extract the frequency widths for a smooth envelope without setting different Thv values, the smooth envelope can be treated as a secondary envelopes, as proposed in [9], and used to automatically extract the frequency feature matrix based on the STMHT technique; this method was successfully applied to detect different types of heart diseases. However, for mitral stenosis and mitral regurgitation noises, the feature matrix was not easily extracted because the second frequency component was missing. Therefore, to improve the classification accuracy 
for diagnosing different types of heart disease and simplify the complexity of the diagnostic method, the smooth envelopes for $C S_{1}$ and $C S_{2}$ extraction in the frequency domain must be considered; additionally, more frequency widths corresponding to different $T h v$ values should be used, and dimensionality reduction should be employed to reduce the number of features considered. Such a classification method could be applied in the efficient extraction of features for diagnosing heart diseases.

Gaussian mixture models (GMMs) have been used in a wide variety of clustering applications $[12,13,14,15,16,17,18]$ due to their powerful mathematical characteristics. Confidence regions are used to diagnose the detection data $\mathbf{x}$ in GMMs, and the optimal confidence regions is determined based on Mahalanobis distance following the Chi-square $\left(\chi^{2}\right)$ distribution. Thus, classifier models with adjustable sizes corresponding to the confidence bounds of the Chi-square $\left(\chi^{2}\right)$ distribution, which can be adjusted by changing the desired confidence level (denoted as $\beta$ ), are proposed. The $\chi^{2}$ confidence bounds used as the classification criteria are employed to diagnose heart diseases.

In summary, this study proposes an innovative and intelligent system. The major contributions in this study are (1) the STMHT-based $C S_{1}$ and $C S_{2}$ are automatically located and extracted; (2) a novel method for obtaining the secondary curves of $C S_{1}$ and $C S_{2}$ are extracted in the frequency domain; (3) frequency features are automatically extracted over the given threshold value; (4) the diagnostic features $\gamma_{1}, \gamma_{2}$ and $\gamma_{3}$ are determined based on PCA; and (5) the confidence region of the $\chi^{2}$ distribution, which are adjusted based on the desired $\beta$, is determined and used as the classification criterion for diagnosing a given HS. The remainder of this paper is organized as follows. Section presents the approach for determining the diagnostic features $\left[\gamma_{1}, \gamma_{2}, \gamma_{3}\right]$, and a definition of the confidence region-based diagnostic method is given. In Section, the performance of the proposed method is compared with that of other efficient methods for diagnosing heart diseases. Finally, the conclusions are provided in Section .

\section{Methodology}

The flow chart of the proposed intelligent system, shown in Fig. 1, consists of three stages: the automatic location and extraction of $C S_{1}$ and $C S_{2}$; the automatic determination of frequency features $\gamma_{1}, \gamma_{2}$ and $\gamma_{3}$; and the establishment of the Mahalanobis distance criterion-based diagnostic method. In stage 1, the STMHT-based curve (denoted as $\mathrm{HS}_{\mathrm{STMHT}}$ ), which is extracted for the $\mathrm{HS}_{\mathrm{E}}$ envelope generated by the HS, is used to segment and extract $C S_{1}$ and $C S_{2}$ from the HS (Fig. $1 \boldsymbol{A}$ ). In stage 2, the envelopes $C S 1_{\mathrm{F}_{\mathrm{E}}}$ and $C S 2_{\mathrm{F}_{\mathrm{E}}}$ for every period $C S 1$ and $C S 2$ are obtained via a novel method, and the frequency features are automatically extracted from $C S_{1 \mathrm{~F}_{\mathrm{E}}}$ and $C S_{2 \mathrm{~F}_{\mathrm{E}}}$ by setting different $T h v$ lines. Finally, the first three principal components, $\gamma_{1}, \gamma_{2}$ and $\gamma_{3}$, which express $86.7 \%$ of the FF information, are determined and used as diagnostic features (Fig. $1 \boldsymbol{B}$ and $\boldsymbol{C}$ ). In stage 3, the GMMbased mixed classification objective function $f_{e t}(\mathbf{x})$ which combines component $k$ with respect to the parameters $\pi_{k}, \mu_{k}$, and $\Sigma_{k}$ and the features $\mathbf{x}=\left[\gamma_{1}, \gamma_{2}, \gamma_{3}\right]$, is generated. Then, the $\chi^{2}$ distribution for component $k$ is determined by calculating the Mahalanobis distance from $\mathbf{x}$ to the class mean $\mu_{k}$ of component $k$, and the adjustable confidence bound (denoted as $\mathrm{MDC}_{k}$ shown in Fig. $1 \boldsymbol{E}$ ) is determined to diagnose heart diseases. 


$$
H S_{\mathrm{E}}[m]=\frac{1}{2 W_{\mathrm{m}}+1} \sum_{k=m-W_{\mathrm{m}}}^{m+W_{\mathrm{m}}}\left(H S_{\mathrm{T}}[k]-\overline{H S}_{\mathrm{T}}[m]\right)^{2}, m=W_{\mathrm{m}}, W_{\mathrm{m}}+1, \ldots M-1-W_{\mathrm{m}},
$$

\section{Stage 1: Automatic location and extraction of $C S_{1}$ and $C S_{2}$}

WD-based heart sound preprocessing

HSs are reported to be primarily dispersed in the frequency range of $20 \sim 700 \mathrm{~Hz}$ $[9,8,2]$. Therefore, according to the sampling frequency (Fs $=44.1 \mathrm{kHz}$ ), WDbased HSs are filtered to obtain the efficient frequency components $(21.5 \sim 689$ $\mathrm{Hz}$ ). The Daubechies wavelet 10 ( $d \mathrm{~B} 10$ ) has been used to give the maximum signalto-noise ratio and minimum root-mean-square error for HSs [19]. Therefore, $d \mathrm{~B} 10$ is selected for use as the mother wavelet for preprocessing HSs. A filtered and normalized sound, denoted as $H S_{\mathrm{T}}$, is shown in Fig. $1 \boldsymbol{A}$.

Extraction of the Viola integral-based heart sound envelope $\left(H S_{\mathrm{E}}\right)$

The Viola integral-based envelope, denoted as $H S_{\mathrm{E}}$, is extracted from the heart sound $H S_{\mathrm{T}}$, as reported in studies [9, 8]; this envelope can effective overcome amplitude variations and complex backgrounds and noise. This concept is described as follows. Consider a filtered sound $H S_{\mathrm{T}}[m]$ for $m=0,1, \ldots, M-1$, where $M$ denotes the number of HSs. In a $W_{\mathrm{m}}$ neighborhood of time $m$, called the width $W_{\mathrm{m}}$ time scale, the $M$-point envelope $H S_{\mathrm{E}}[m]$ is obtained by Eq. (1): where

$$
\overline{H S}_{\mathrm{T}}[m]=\frac{1}{2 W_{\mathrm{m}}+1} \sum_{k=m-W_{\mathrm{m}}}^{m+W_{\mathrm{m}}} H S_{\mathrm{T}}[k]
$$

$W_{\mathrm{m}}=2205$ if the duration of $C S_{1}$ or $C S_{2}$ greater than 0.13 s. Finally, normalization is performed by setting the maximum amplitude of $H S_{\mathrm{E}}$ to 1 (Fig. $1 \boldsymbol{A}$ ).

STMHT extraction for $\mathbf{H S}$ (denoted as $H S_{\mathrm{STMHT}}$ )

Given an $M$-point HS, the STMHT for the HSs , $H S_{\mathrm{STMHT}}$, is computed from Eq. (3) where $n=(N-1) / 2, \ldots, M-1-(N-1) / 2$, and $W_{N}[l](l=-(N-$ $1) / 2, \ldots,(N-1) / 2)$ is a moving window of odd length $N$. According to studies $[8,2]$, the length $N$ is set to 44101 .

Automatic extraction of $\mathrm{HS}_{\mathrm{STMHT}}$-based $C S_{1}$ and $C S_{2}$

The characteristics of $H S_{\text {STmht }}$ considered in studies [8, 2], as shown in Fig. $2 \boldsymbol{A}$ and $\boldsymbol{C}$, are as follows:

1) The negative-to-positive $(\mathbf{N 2 P})$ points of $H S_{\mathrm{STMHT}}$, denoted by $\boldsymbol{\Delta}$, correspond to the geometry center peaks of $S_{1}$ and $S_{2}$.

2) The geometry center between $S_{1}$ and $S_{2}$, denoted by $\nabla$ is determined by the positive-to-negative $\mathbf{P} 2 \mathbf{N}$ points of $H S_{\text {STMHT }}$.

The interval from $S_{2}$ to $S_{1}$ is generally greater than that from $S_{1}$ to $S_{2}$ in one period of an HS $[20,21,22]$. Therefore, the $\mathbf{N 2 P}$ and $\mathbf{P} 2 \mathbf{N}$-based $C S_{1}$ and $C S_{2}$ features can be automatically segmented from one period of an HS and extracted by two procedures, as described in the following section. 


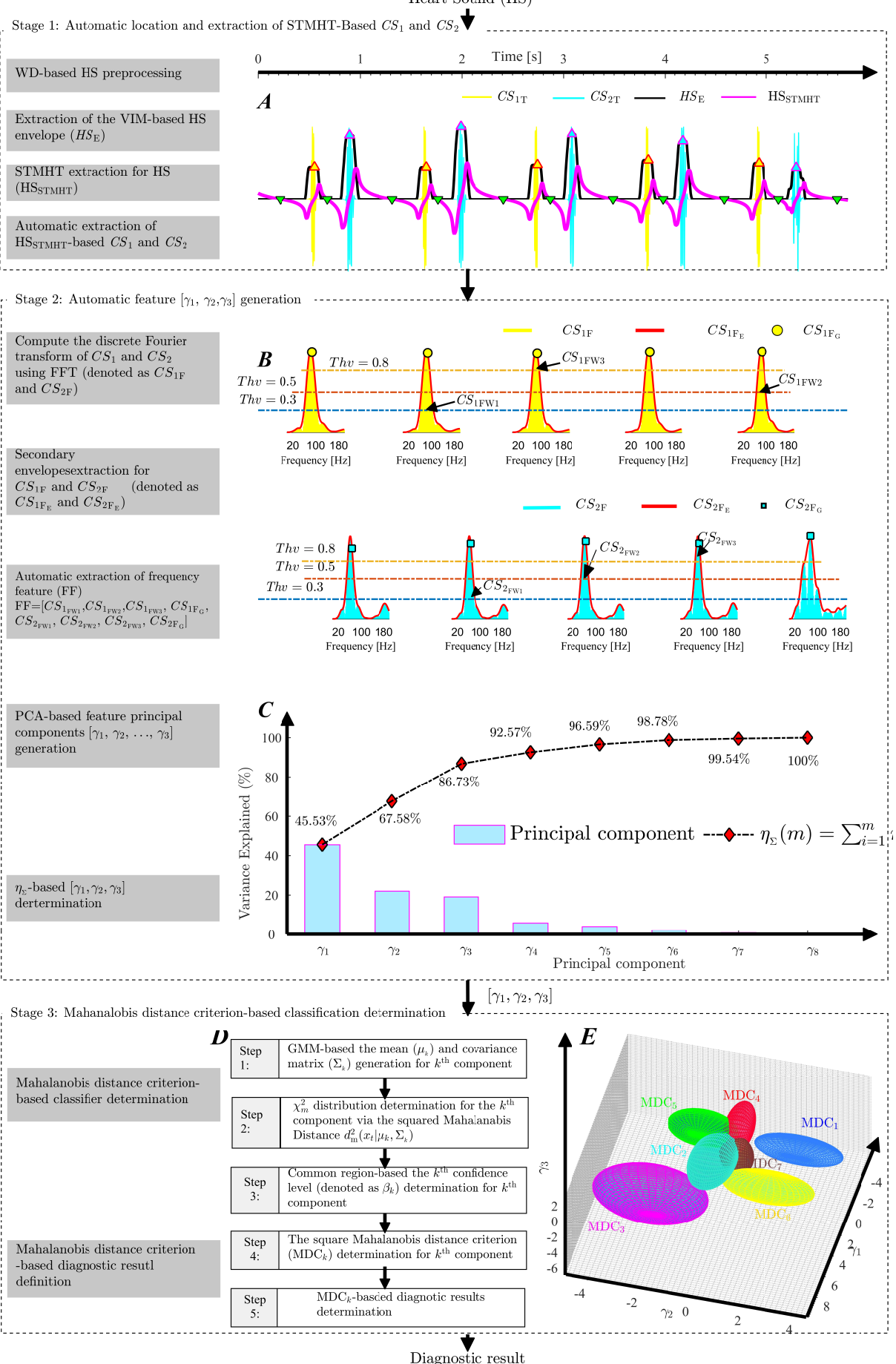

Fig. 1. Flow chart of the proposed methodology.

$$
\begin{gathered}
\operatorname{HS}_{\mathrm{STMht}}[n]=\sum_{m=n-\frac{N-1}{2}}^{n+\frac{N-1}{2}} H S_{\mathrm{E}}[m] W_{N}[m-n] W_{\mathrm{E}}\left[m-\left(n-\frac{N-1}{2}\right)\right], \\
W_{\mathrm{E}}[i]= \begin{cases}\frac{\cos \left(\frac{N-1-2 i}{2 N} \pi\right)-\cos \left(\frac{N-1-2 i}{2} \pi\right)}{N \sin \left(\frac{N-1-2 i}{2 N} \pi\right)} & \text { for } i=0,1, \ldots, N-1 . \\
0 & \text { for } i=\frac{N-1}{2}\end{cases}
\end{gathered}
$$




$$
\begin{aligned}
& D S_{H S_{\mathrm{STMHT}}}[i]=S_{H S_{\mathrm{STMHT}}}[i+1]-S_{H S_{\mathrm{STMHT}}}[i], i=1,2, \ldots, n . \\
& D_{\mathrm{N} 2 \mathrm{P}}[i]=\mathrm{N} 2 \mathrm{P}[i+1]-\mathrm{N} 2 \mathrm{P}[i], i=1,2, \ldots, n .
\end{aligned}
$$

\section{(a) N2P and P2N location}

The algorithm for detecting N2P and P2N is detailed as follows.

1) First, the signum function of $H S_{\mathrm{STMHT}}$, denoted as $S_{H S_{\mathrm{STMHT}}}$, is calculated by

$$
S_{H S_{\mathrm{STMHT}}}=\left\{\begin{array}{lll}
-1 & \text { if } & H S_{\mathrm{STMHT}}<0 \\
0 & \text { if } & H S_{\mathrm{STMHT}}=0 \\
+1 & \text { if } & H S_{\mathrm{STMHT}}>0
\end{array}\right.
$$

2) Then, the variation in $S_{H S_{\text {Sтмнт }}}\left(D S_{H S_{\text {Sтмнт }}}\right)$ is determined from Eq. (6)

3) Finally, $\mathbf{N 2 P}$ and $\mathbf{P} \mathbf{2 N}$ are determined by

$$
\left\{\begin{array}{lll}
\mathrm{N} 2 \mathrm{P}=i / F_{\mathrm{S}} & \text { if } & D S_{H S_{\mathrm{STMht}}}[i]=+2 \\
\mathrm{P} 2 \mathrm{~N}=i / F_{\mathrm{S}} & \text { if } & D S_{H S_{\mathrm{STMHT}}}[i]=-2
\end{array}\right.
$$

(b) Automatic extraction of $C S_{1}$ and $C S_{2}$

1) Calculate the difference between two adjacent $\mathbf{N 2 P}$, denoted as $D_{\mathrm{N} 2 \mathrm{P}}$, with Eq. (8)

2) Determine the points $C S_{12}$ and $C S_{21}$ that are used for segmentation from $C S_{1}$ to $C S_{2}$ and from $C S_{2}$ to $C S_{1}$, respectively, by using Eq. (9).

3) Extract $C S_{1}$ (denoted as $C S_{1 i}$ ) and $C S_{2}$ (denoted as $C S_{2 i}$ ) for the $i$ th period of an HS as follows

$$
\left\{\begin{array}{l}
C S_{1_{i}}=H S_{\mathrm{T}}\left[C S_{21_{i}}: C S_{12_{i}}\right] \\
C S_{2 i}=H S_{\mathrm{T}}\left[C S_{12_{i}}: C S_{21_{i+1}}\right]
\end{array}\right.
$$

The automatic extraction procedures for $C S_{1}$ and $C S_{2}$ are illustrated in Fig. 2. Fig. $2(\boldsymbol{A}$ and $\boldsymbol{B})$ show a typical AR sound, and the typical NM sound is shown in Fig. $2(\boldsymbol{C}$ and $\boldsymbol{D})$.

\section{Stage 2: Automatic feature generation}

Secondary envelopes for $C S_{1}$ and $C S_{2}$ definition

Given an $M$-point HS, the secondary envelope in the frequency-domain, denoted as $H S_{\mathrm{F}}$, can be calculated from Eq. (11): where $l_{1}, l_{2}$ and $H S_{\mathrm{F}}[k]$ are defined by Eq. (12): $|\cdot|$ is the absolute value sign, $2 L_{1}+1$ is the first window width, and $2 L_{2}+1$ is the second window width. According to studies $[9,25], L_{1}$ and $L_{2}$ are set to 9 and 17 , respectively. Moreover, $H S_{F_{\mathrm{E}}}$ is also normalized by setting the maximum amplitude of $H S_{F_{\mathrm{E}}}$ to 1 .

$$
\begin{cases}C S_{21}[i]=\mathrm{P} 2 \mathrm{~N}[i], \mathrm{CS}_{12}[i]=\mathrm{P} 2 \mathrm{~N}[i+1], & \text { if } \quad D_{\mathrm{N} 2 \mathrm{P}}[i]<D_{\mathrm{N} 2 \mathrm{P}}[i+1] \\ C S_{21}[i]=\mathrm{P} 2 \mathrm{~N}[i+1], \mathrm{CS}_{12}[i]=\mathrm{P} 2 \mathrm{~N}[i+2], & \text { Otherwise }\end{cases}
$$




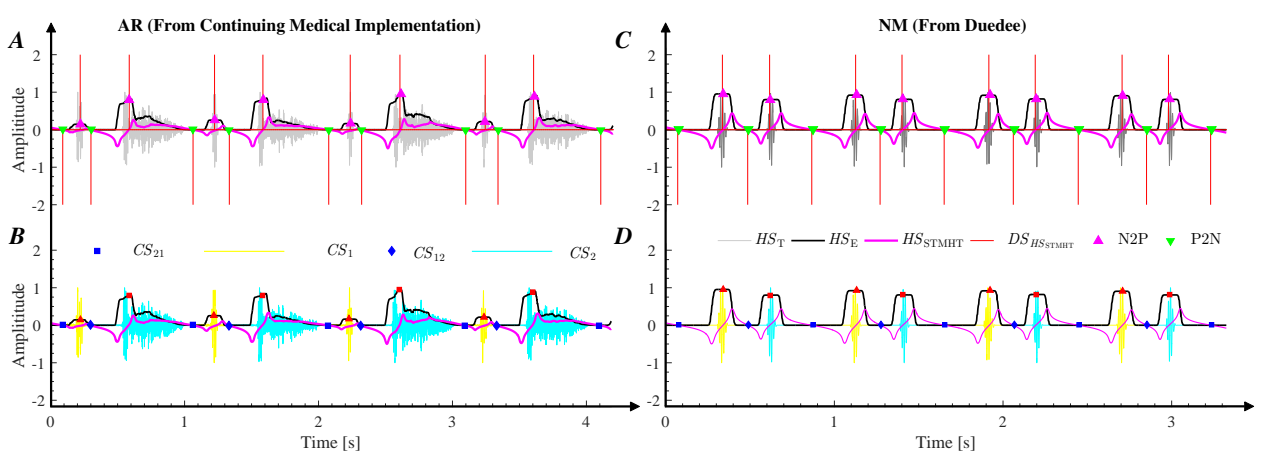

Fig. 2. The automatic extraction procedures for $C S_{1}$ and $C S_{2} \cdot \boldsymbol{A}-\boldsymbol{B}$ show the procedure for an example of a typical AR from the database in [23]. $\boldsymbol{C}-\boldsymbol{D}$ show the procedure for an example of a typical normal sound from [24].

$$
H S_{F_{\mathrm{E}}}[k]=\frac{\sum_{l_{1}}\left(L_{1}+L_{2}+1-\left|l_{1}\right|\right) H S_{\mathrm{F}}\left[k+l_{1}\right]-\sum_{l_{2}} H S_{\mathrm{F}}\left[k+l_{2}\right]}{\left(2 L_{1}+1\right)\left(2 L_{2}+1\right)},
$$

The secondary envelopes for $C S_{1}$ and $C S_{2}$, denoted as $C S_{1 F_{\mathrm{E}}}$ and $C S_{2 F_{\mathrm{E}}}$ respectively, are illustrated by using an example of a normal sound in Fig. 3. $C S_{1}$ (colored yellow) and $C S_{2}$ (colored cyan), which are first automatically extracted based on the STMHT algorithm, are shown in Fig. $3 \boldsymbol{A}$. Then, the frequency components of $C S_{1}$ and $C S_{2}$ are calculated with Eq. (12) and plotted in Fig. 3(B and $\left.\boldsymbol{C}\right)$. Finally, the secondary envelopes for $C S_{1}$ and $C S_{2}$, denoted as $C S_{1 F_{\mathrm{E}}}$ and $C S_{2 F_{\mathrm{E}}}$ respectively, are extracted from Eq. (11), and the results are shown in Fig. 3( $\boldsymbol{B}$ and $\boldsymbol{C})$. In the following subsection, the frequency features for $C S_{1}$ and $C S_{2}$ are defined and automatically extracted by using the threshold line method.

Definition and automatic extraction of secondary envelope-based frequency features The frequency features are illustrated in Fig. $3(\boldsymbol{B}$ and $\boldsymbol{C})$, and their gravities are calculated by

$$
\left\{\begin{array}{l}
C S_{1 \mathrm{G}}=\frac{\sum_{k=0}^{M-1} k \times C S_{1 F_{\mathrm{E}}}[k]}{\sum_{k=0}^{M-1} C S_{1 F_{\mathrm{E}}}[k]} \\
C S_{2 \mathrm{G}}=\frac{\sum_{k=0}^{M-1} k \times C S_{2 F_{\mathrm{E}}}[k]}{\sum_{k=0}^{M-1} C S_{2 F_{\mathrm{E}}}[k]}
\end{array},\right.
$$

The frequency widths over a given threshold value are defined and calculated by

$$
\left\{\begin{array}{l}
C S_{1 \mathrm{FWi}}=R_{p_{i}}-L_{p_{i}} \\
C S_{2 \mathrm{FWi}}=R_{p_{i}}-L_{p_{i}}
\end{array} \quad, i=1,2,3\right.
$$

where $L_{p_{i}}$ and $R_{p_{i}}$ are the $i^{\text {th }}$ left and right intersections, respectively, of $C S_{1 F_{\mathrm{E}}}$ and $C S_{2 F_{\mathrm{E}}}$ over the Thv lines (Thv=0.3, 0.5 and 0.8). Moreover, the frequency features are expressed based on Eq. (15).

$$
\left\{\begin{array}{l}
l_{1}=-\left(L_{1}+L_{2}\right),-\left(L_{1}+L_{2}\right)+1, \ldots,\left(L_{1}+L_{2}\right) \\
l_{2}=-\left(L_{1}-L_{2}-1\right),-\left(L_{1}-L_{2}-1\right)+1, \ldots,\left(L_{1}-L_{2}-1\right) \\
H S_{\mathrm{F}}[k]=\left|\sum_{m=0}^{\mathrm{M}-1} H S_{T}[m+1] e^{\left(-j \frac{2 \pi k l}{M}\right)}\right|, \quad k=0,1,2, \ldots, M-1
\end{array},\right.
$$




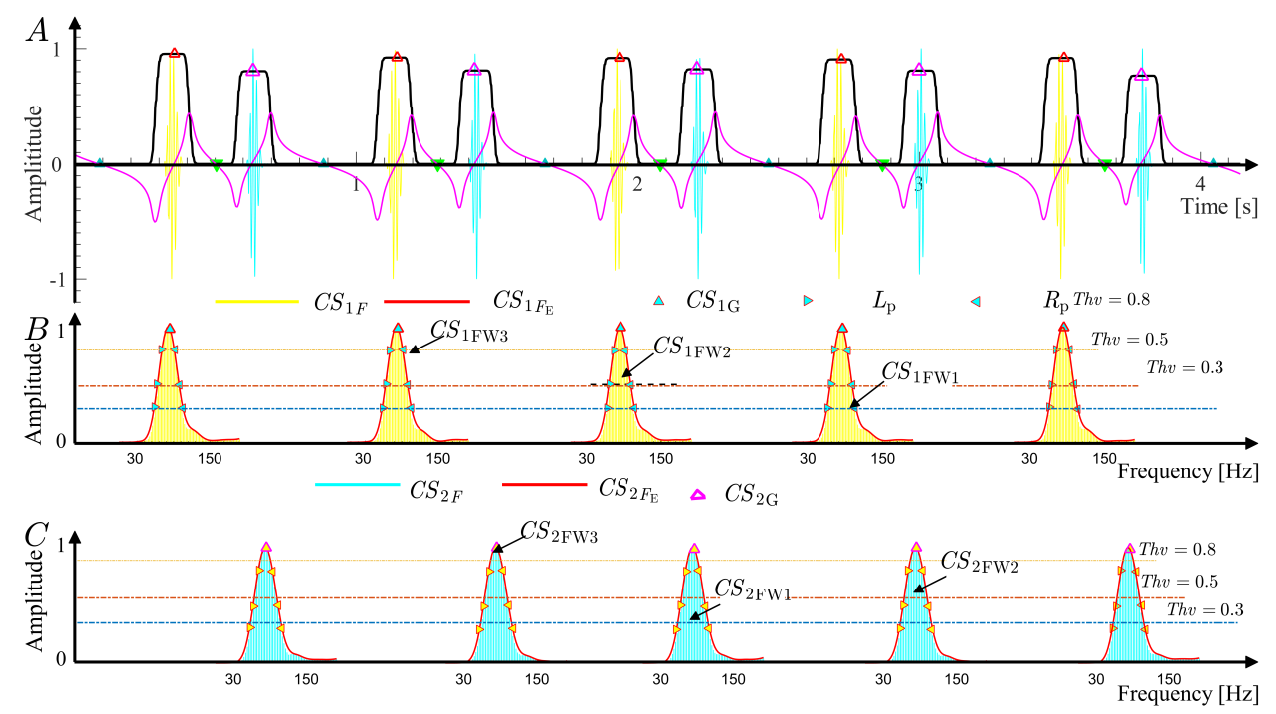

Fig. 3. Example of feature definition and automatic extraction.

$\mathrm{FF}=\left[C S_{1 \mathrm{FW} 1}, C S_{1 \mathrm{FW} 2}, C S_{1 \mathrm{FW} 3}, C S_{1 \mathrm{G}}, C S_{2 \mathrm{FW} 1}, C S_{2 \mathrm{FW} 2}, C S_{2 \mathrm{FW} 3}, C S_{2 \mathrm{G}}\right]$

Experimental results for several typical types of heart disease

The features FF of six six typical and normal sounds are illustrated in Fig. 4. From Fig. 4, $C S_{1}$ and $C S_{2}$ are first automatically located and extracted, as shown in Fig. $4 \boldsymbol{A}$; then, the envelopes for every $C S_{1}$ and $C S_{2}$ are extracted by Eq. (11), as shown in Fig. $4 \boldsymbol{B}$ and Fig. $4 \boldsymbol{C}$, respectively. Finally, the features defined by Eq. (15) for $C S_{1}$ and $C S_{2}$ in the frequency domain are automatically extracted with Eqs. (1314). The experimental sounds are 665-period AR sounds (3M database [26], medical sound library [27], heart auscultation sounds [28], auscultation sound [29], continuing medical implementation [23], sounds Database of the University of Dundee [24], and patients only with AR disease from the Nanyang First People's Hospital), 381period AS sounds (continuing medical implementation [23], sounds database of the University of Dundee [24], 3M database [26], medical sound library [27], auscultation sound [29], and patients only with AS disease from the Nanyang first People's Hospital, and heart auscultation sounds [28]), 315-period ASD sounds (Medical sound library [27], heart auscultation sounds [28], 3M database [26], patients only with ASD disease from the Nanyang First People's Hospital, and medical sound library [27]), 769-period MR sounds (3M database [26], sounds database of the University of Dundee [24], heart auscultation sounds [28], medical sound library [27], and auscultation sound [29]), 439-period MS sounds(3M database [26], auscultation sound [29], medical sound library [27], and continuing medical implementation [23]), and 1056-period NM sounds(3M database [26], Michigan database [30], medical sound library [27], ThinkLabs database [31], and healthy undergraduates from Nanyang Institute of Technology, China)(whom I thank for the data used in this study)). Moreover, the boxplots for the features are plotted in Fig. 5 , where Fig. $5 \boldsymbol{A}$ shows the features extracted from $C S_{1}$ and Fig. $5 \boldsymbol{B}$ shows the features from $C S_{2}$ for each type of heart disease. The scatter plots of features in Fig. 5 illustrate the 
discrimination ability of the model in distinguishing among different heart diseases and highlighting the following findings:

W The MS and VSD sounds are easy to distinguish from the other sounds by using $C S_{1 \mathrm{FW} 1}$ (Fig. $5 \boldsymbol{A}$ ).

By using the $C S_{2 \mathrm{FW} 1}($ Fig. $5 \boldsymbol{B})$, the VSD sound is easy to distinguish from the other sounds.

The MS sound is easy to distinguish from the other sounds based on $C S_{1 \mathrm{FW} 2}$ (Fig. 5A).

By using the $C S_{2 \mathrm{FW} 2}$ (Fig. $5 \boldsymbol{B}$ ), the AR and VSD sounds are distinguished from other sounds.

The NM sound is easy to distinguish from other sounds using $C S_{1 \mathrm{FW} 3}$ (Fig. $5 \boldsymbol{A})$.

W The AR and VSD sounds are easy to distinguish from the other sounds using $C S_{2 \mathrm{FW} 3}$, as shown in Fig. $5 \boldsymbol{B}$.

$\boldsymbol{A}$ Fig. $5 \boldsymbol{A}$ indicates that $C S_{1 \mathrm{G}}$ can be used to easily distinguish $\mathbf{M R}$ from other sounds and the AS and ASD sounds from other sounds.

$\boldsymbol{A}$ Fig. $5 \boldsymbol{B}$ shows that the distribution of $C S_{2 \mathrm{G}}$ from AS sounds is different from that for other sounds, except $\mathbf{N M}$ sounds. Moreover, $C S_{2 \mathrm{G}}$ can be used to easily distinguish different sounds.

The analysis results discussed above indicate that different combinations of several features defined by Eq. (15) can be used to distinguish among various types of heart disease. Therefore, to simplify features and develop a diagnostic method that is simple and effective, dimension reduction is used to determine new features; this process is described in detail as follows.

\section{Diagnostic feature determination}

To simplify the computation when using features to diagnose heart diseases, PCA, a linear dimensionality reduction technique for finding principal components and replacing high-dimension data, is employed. PCA has been used in studies of heart arrhythmias classification [32], heart disease classification[33, 2], emotion recognition [34], respiratory rate extraction [35], electrocardiogram heart disease diagnosis [36], and real-time magnetoencephalography neurofeedback [37]. A few major principal components are used to characterize $\mathbf{H S}$ features and diagnose heart diseases. The flow chart corresponding to the new features that are generated via PCA for FF is shown in Fig. 6. The eigenvector $\xi_{i}$, which corresponds to the eigenvalue $\lambda_{i}$ and is calculated for the matrix $Z$ in step 2 , as shown in Table 2 , is the actual weighted coefficient for the $i$ th principal component $\gamma_{i}$. Table 2 shows that the largest absolute coefficients in the first principal component $\gamma_{1}$ are $C S_{1 \mathrm{FW} 1}, C S_{2 \mathrm{FW} 2}$ and $C S_{1 \mathrm{FW} 2}$; the second principal component $\gamma_{2}$ is mainly weighted based on $C S_{1 \mathrm{G}}, C S_{2 \mathrm{FW} 2}$, $C S_{1 \mathrm{FW} 1}$ and $C S_{2 \mathrm{FW} 3}$; and the third component $\gamma_{3}$ is mainly weighted based on $C S_{1 \mathrm{FW} 2}, C S_{1 \mathrm{FW} 3}$ and $C S_{2 \mathrm{FW} 3}$ (Table 2). To determine the smallest number of principal components $m$ should be considered, the Pareto chart is used; this chart provides a tool for visualizing the Pareto principle, which states that observing a small set of variables that influence a common outcome is more common than detecting many variables that influence the same outcome. This approach has been used to determine the percent variability explained by each principal component 

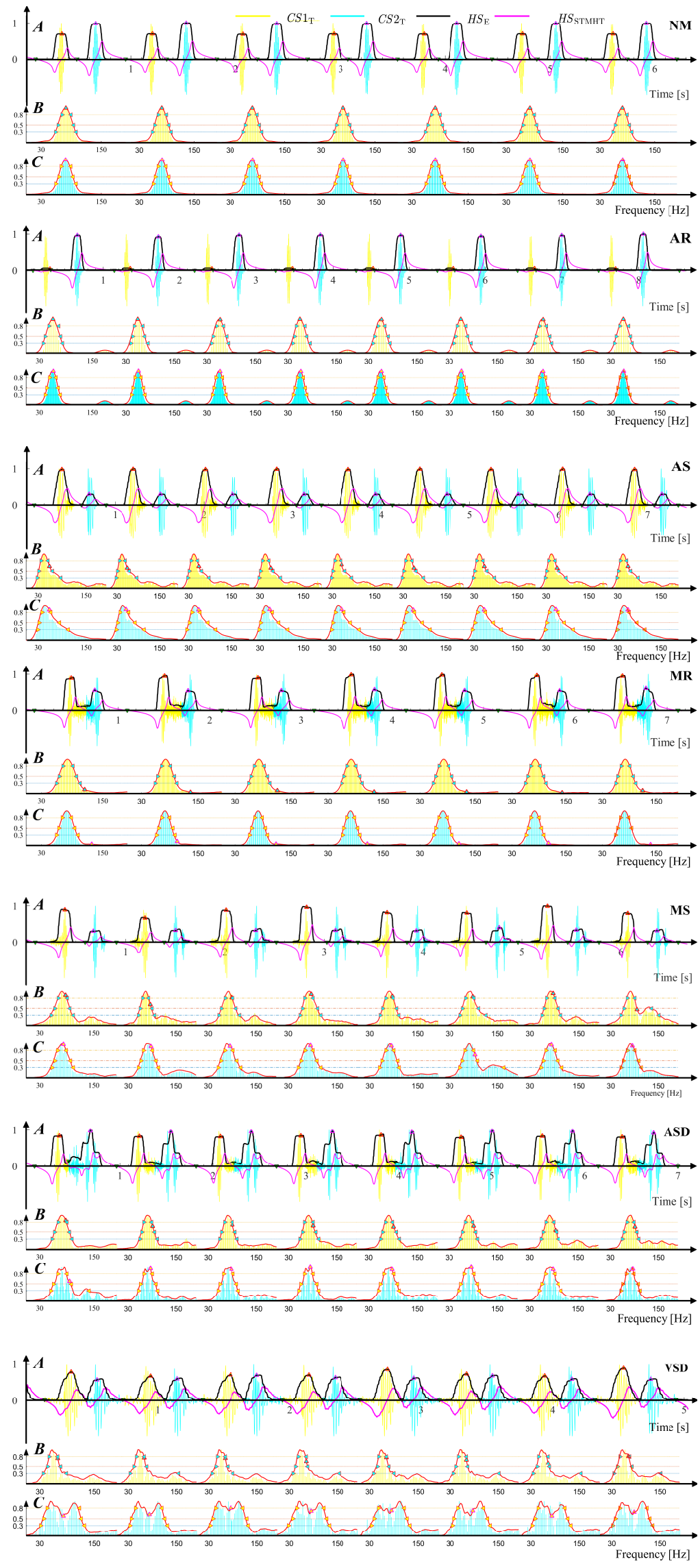

Fig. 4. Examples of a typical normal sound and six types of typical heart disease sounds. 


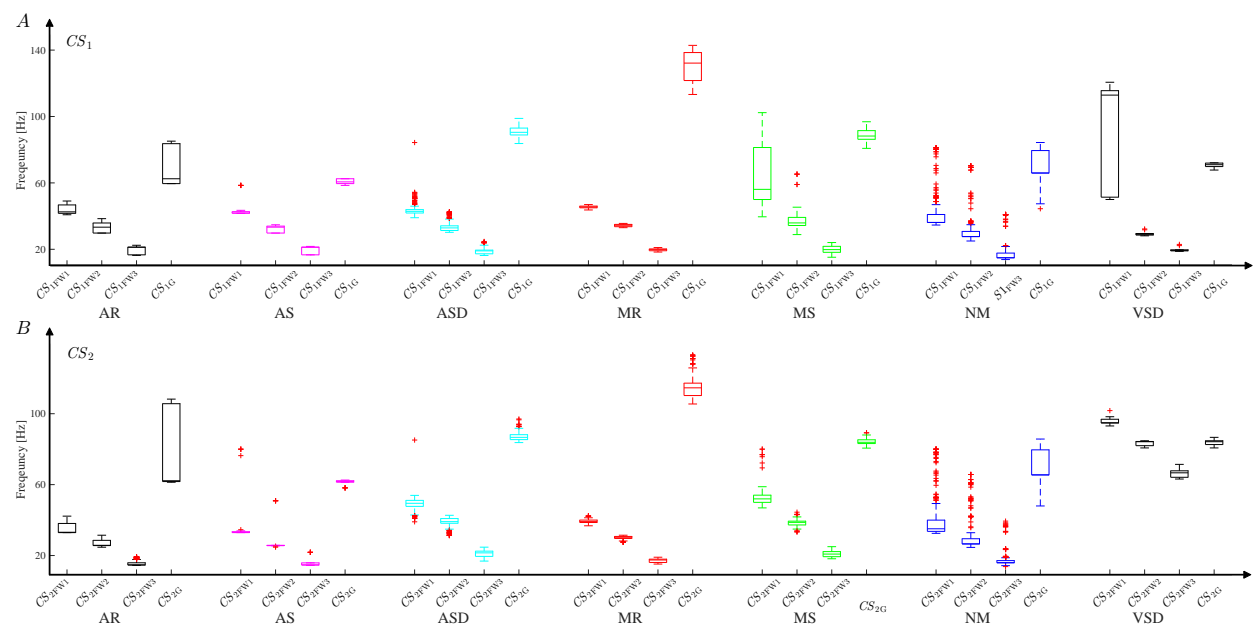

Fig. 5. Box plot representation of FF for each type of heart disease. $\boldsymbol{A}$ shows the box plots for features from $C S_{1}$. In addition, $\boldsymbol{B}$ represents the features from $C S_{2}$.

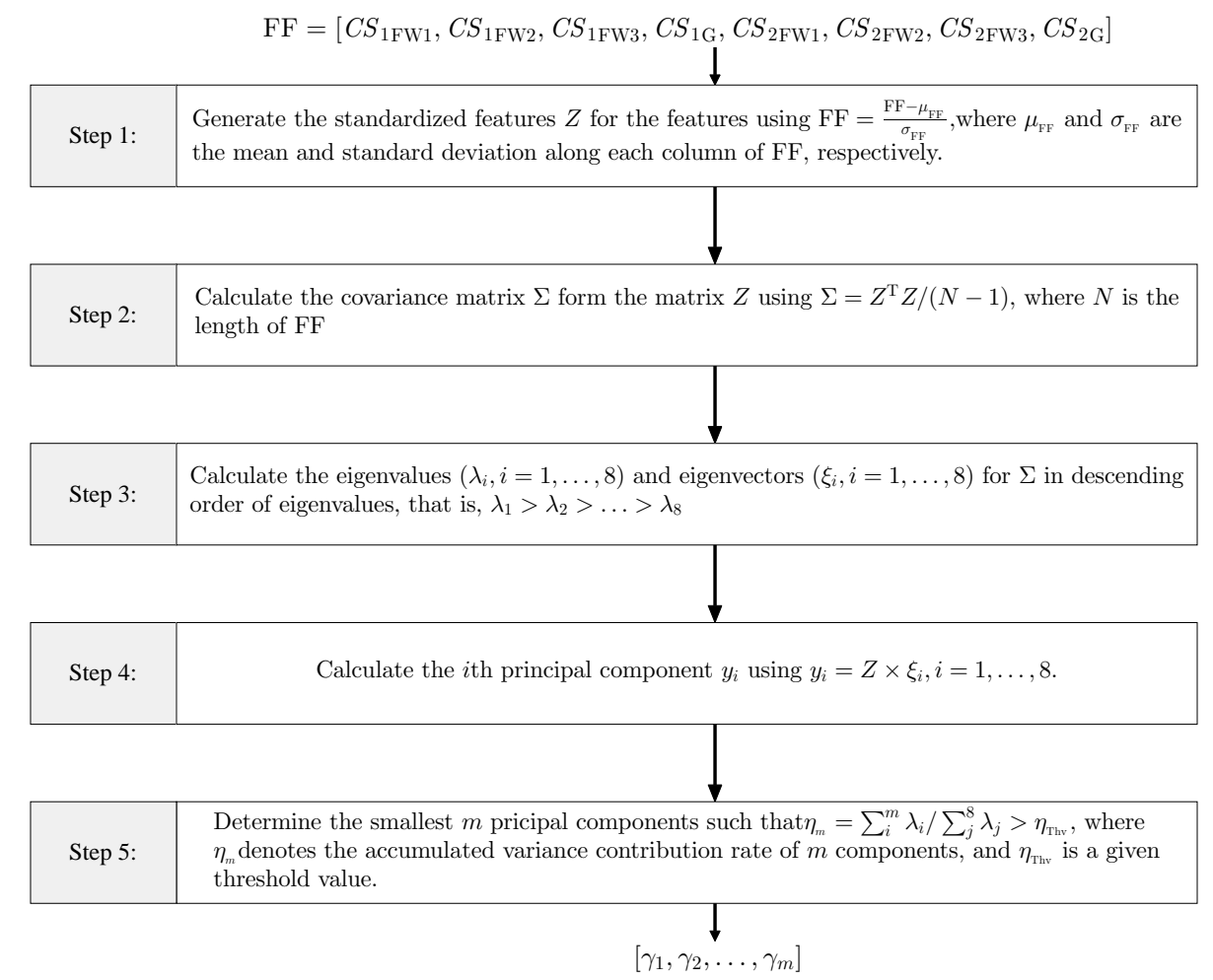

Fig. 6. The flow chart corresponding to the efficient feature generation procedure. 
Table 1. Mean $\left(\mu_{\mathrm{FF}}\right)$ and standard deviation $\left(\sigma_{\mathrm{FF}}\right)$ of the features.

\begin{tabular}{|c|c|c|c|c|c|c|c|c|}
\hline \multirow{3}{*}{ Statistics } & \multicolumn{8}{|c|}{ Frequency features $\left(\mu_{\mathrm{FF}}+\sigma_{\mathrm{FF}}\right)$} \\
\hline & \multicolumn{4}{|c|}{ Features from $C S_{1}$} & \multicolumn{4}{|c|}{ Features from $\mathrm{CS}_{2}$} \\
\hline & $C S_{1 \mathrm{FW} 1}$ & $C S_{1 \mathrm{FW} 2}$ & $C S_{1 \mathrm{FW} 3}$ & $C S_{1 \mathrm{G}}$ & $C S_{2 \mathrm{FW} 1}$ & $C S_{2 \mathrm{FW} 2}$ & $C S_{2 \mathrm{FW} 3}$ & $C S_{2 \mathrm{G}}$ \\
\hline$\mu_{\mathrm{FF}} \pm \sigma_{\mathrm{FF}}$ & $45.3 \pm 11.8$ & $33.1 \pm 5.8$ & $18.8 \pm 3.6$ & $80.6 \pm 21.7$ & $44.1 \pm 23.1$ & $32.2 \pm 9.1$ & $18.4 \pm 6.5$ & $79.8 \pm 18.9$ \\
\hline
\end{tabular}

Table 2. Eigenvector $\xi_{i}$ and eigenvalue $\lambda_{i}(i=1, \ldots, 8)$ for $\Sigma$ in descending order of eigenvalues

\begin{tabular}{lcccccccc}
\hline \multirow{2}{*}{ Features } & \multicolumn{7}{c}{ Eigenvector (eigenvalue) in descending order of eigenvalues } \\
\cline { 2 - 8 } & $\xi_{1}\left(\lambda_{1}=3.6423\right)$ & $\xi_{2}\left(\lambda_{2}=1.7643\right)$ & $\xi_{3}\left(\lambda_{3}=1.5316\right)$ & $\xi_{4}\left(\lambda_{4}=0.4671\right)$ & $\xi_{5}\left(\lambda_{5}=0.3226\right)$ & $\xi_{6}\left(\lambda_{6}=0.1748\right)$ & $\xi_{7}\left(\lambda_{7}=0.0606\right)$ & $\xi_{8}\left(\lambda_{8}=0.0368\right)$ \\
\hline$S 1_{\mathrm{FW} 1}$ & 0.4309 & -0.2169 & 0.0818 & -0.2179 & 0.8062 & -0.2394 & -0.0499 & 0.0616 \\
$S 1_{\mathrm{FW} 2}$ & 0.3716 & -0.0757 & 0.5303 & 0.0431 & -0.0306 & 0.6778 & 0.2873 & -0.1735 \\
$S 1_{\mathrm{FW} 3}$ & 0.3411 & -0.0983 & 0.5431 & 0.0444 & -0.4364 & -0.6126 & -0.1001 & -0.0375 \\
$S 1_{\mathrm{G}}$ & 0.2385 & 0.6501 & -0.0157 & -0.2122 & 0.0212 & 0.0986 & -0.5500 & -0.4031 \\
$S 2_{\mathrm{FW} 1}$ & 0.3313 & 0.0487 & -0.2471 & 0.8958 & 0.0977 & -0.0299 & -0.0699 & -0.0944 \\
$S 2_{\mathrm{FW} 2}$ & 0.4475 & -0.2390 & -0.2924 & -0.1628 & -0.3044 & 0.2633 & -0.3928 & 0.5607 \\
$S 2_{\mathrm{FW} 3}$ & 0.3517 & -0.2191 & -0.5142 & -0.2650 & -0.2357 & -0.1047 & 0.3750 & -0.5353 \\
$S 2_{\mathrm{G}}$ & 0.2632 & 0.1026 & -0.0768 & -0.0662 & -0.0207 & -0.1308 & 0.5506 & 0.4386 \\
\hline
\end{tabular}

(Fig. $7 \boldsymbol{A}$ ). Therefore, according to the smallest $m$ value such that $\eta_{\Sigma}(m)>80 \%$ [38], combined with the scatter plot for the first $m$ principal components, the smallest $m$ is determined. The Pareto chart of the PCA results in Fig. $7 \boldsymbol{A}$ shows the explained variance and accumulated variance for each principal component $\gamma_{i}$, where $i=1,2, \ldots, 8$. According to Fig. $7 \boldsymbol{A}, 67.58 \%$ of the total variance is captured by the first two components, $\gamma_{1}$ and $\gamma_{2}$, and $86.73 \%$ of the total variance is captured by the first three components $\gamma_{1}, \gamma_{2}$ and $\gamma_{3}$. Therefore, the following conclusions can be obtained.

(a) $\gamma_{1}$ and $\gamma_{2}$ lead to a dimensionality reduction of $75 \%$ (from 8 to 2 variables) and only $32.42 \%$ information loss. The scatter diagram of $\gamma_{1}$ and $\gamma_{2}$ given in Fig. $7 \boldsymbol{B}$ indicates that although the distribution region corresponding to each type of heart disease is obviously different and the overlaps between $\mathbf{M R}$ and other diseases, AR and other diseases, and VSD and other diseases are small, the overlaps among MS, ASD, NM, and AS are relatively large; therefore, it is difficult to accurately distinguish among these four types of heart diseases.

(b) However, the scatter diagram of $\gamma_{1}, \gamma_{2}$ and $\gamma_{3}$, plotted in Fig. $7 \boldsymbol{C}$, shows that there are different distribution regions for these types of heart diseases. In addition, $\eta_{\Sigma}(3)=86.73 \%$, as shown in Fig. $7 \boldsymbol{A}$, based on feature number determination [38]. Thus, $\gamma_{1}, \gamma_{2}$ and $\gamma_{3}$ lead to a dimensionality reduction of $62.5 \%$ (from 8 to 3 variables) with only $13.27 \%$ information loss. The scatter diagram of $\gamma_{1}, \gamma_{2}$ and $\gamma_{3}$ in Fig. $7 \boldsymbol{C}$ is used to verify the different distribution regions corresponding to these types of heart diseases.

Therefore, $m$ is set to 3 , and the new 3-dimensional feature matrices consisting of $\gamma_{1}, \gamma_{2}$ and $\gamma_{3}$ (see Fig. $7 \boldsymbol{C}$ ) are used to diagnose heart diseases.

Stage 3: Classification based on the squared Mahalanobis distance criterion Classifier determination

The squared Mahalanobis distance classification criterion-based diagnostic methodology, consisting of the five sequential steps as shown in the flow chart (Fig. 8A ), is proposed to diagnose HSs and is described in the following 5 steps.

Step 1. GMM-based $\mu_{k}$ and $\Sigma_{k}$ generation

In the design step of GMM, the estimated target function, $f_{e t}(\mathbf{x})$, is a mixture of $d$-dimensional normal Gaussian distributions $p\left(\mathbf{x} \mid \mu_{k}, \Sigma_{k}\right)$ that reflect the training 

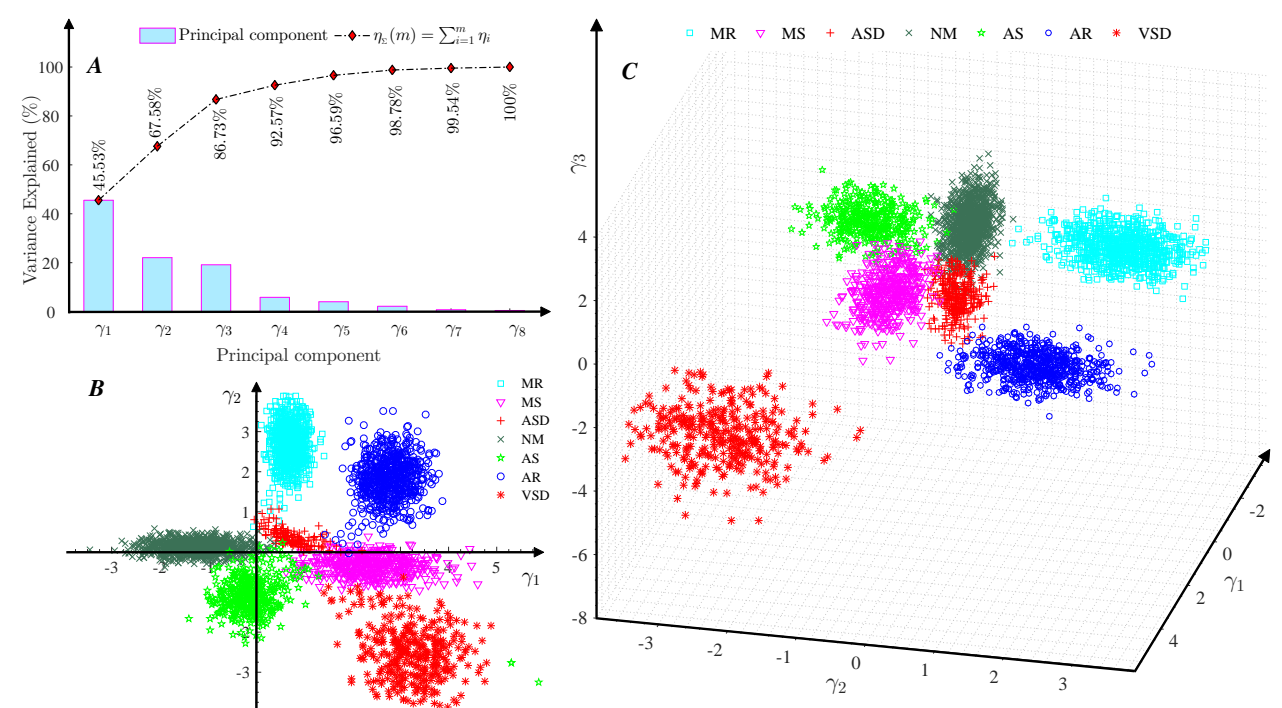

Fig. 7. PCA results. $\boldsymbol{A}$ shows the Pareto chart of the variance by contribution of each principal component, $\boldsymbol{B}$ plots the scatter diagram of the first two components $\gamma_{1}$ and $\gamma_{2}$, and $\boldsymbol{C}$ shows the scatter diagram of the first three components $\gamma_{1}, \gamma_{2}$ and $\gamma_{3}$.

pattern of each component; it is assumed that components can be modeled by mixtures of normal Gaussian distributions by

$$
f_{e t}(\mathbf{x})=\sum_{k=1}^{K} \pi_{k} p\left(\mathbf{x} \mid \mu_{k}, \Sigma_{k}\right)
$$

where

$$
p(\mathbf{x})=\frac{1}{\sqrt{(2 \pi)^{d}\left|\Sigma_{k}\right|}} \exp ^{\left(-\frac{1}{2}\left(\mathbf{x}-\mu_{k}\right)^{\mathrm{T}} \Sigma_{k}^{-1}\left(\mathbf{x}-\mu_{k}\right)\right)}
$$

expresses the posterior probabilities corresponding to each component; $K$ is the number of components; $\pi_{k}$ corresponds to the mixed weights, such that $\sum_{k=1}^{K} \pi_{k}=$ 1 ; and $\mu_{k}$ and $\Sigma_{k}$ are the mean value and covariance matrix of the $k^{\text {th }}$ component, respectively. Because the goal is to maximize the function $f_{e t}(\mathbf{x})$, the parameters $\left(\pi_{k}, \mu_{k}\right.$, and $\left.\Sigma_{k}\right)$ are determined based on the EM algorithm [39] for a set of sample records. Based on the types of heart disease described in Subsection and the scatter diagram plotted in Fig. $7 \mathbf{C}$, the number of Gaussian mixture components is set to $K=7$, and the fitgmdist function in MATLAB 2018b is used to return a GMM with $K=7$ components fitted to the features $\left[\gamma_{1}, \gamma_{2}, \gamma_{3}\right]$ established in Section using the EM algorithm by assigning a posterior probability to each component density with respect to each observation. Furthermore, the regularization value is set as 0.01 to avoid ill-conditioned covariance estimates, and the number of optimization iterations is set to 1000 based on experience. The Gaussian mixture parameter estimates for $\pi_{k}, \mu_{k}$ and $\Sigma_{k}$ are obtained and shown in Table 3. To characterize the 3 -dimensional interspace corresponding to each 3-dimensional Gaussian component for diagnosing heart diseases, the 3-dimensional interspaces can be used as 3-dimensional classifiers to diagnose heart diseases with high classification accuracy; the overlapping interspace between two random components is made as small 
Table 3. The Gaussian mixture parameter estimates are achieved for the new features $\left[\gamma_{1}, \gamma_{2}, \gamma_{3}\right]$ by setting the number of Gaussian mixture components as 7 .

\begin{tabular}{|c|c|c|c|c|c|c|c|c|}
\hline \multirow{3}{*}{ Components } & \multirow{3}{*}{ Component number } & \multicolumn{6}{|c|}{ Gaussian mixture parameter estimates } & \\
\hline & & \multirow[t]{2}{*}{$\pi_{k}$} & \multicolumn{3}{|c|}{$\mu_{k}$} & \multicolumn{3}{|c|}{$\Sigma_{k}$} \\
\hline & & & & & & 0.0425 & -0.0007 & 0.0013 \\
\hline \multirow[t]{3}{*}{ MR Classifier } & $k=1$ & 0.1947 & 0.7056 & 2.7126 & 1.4950 & -0.0007 & 0.2343 & -0.0126 \\
\hline & & & & & & 0.0013 & -0.0126 & 0.2122 \\
\hline & & & & & & 0.3310 & -0.0094 & -0.0122 \\
\hline \multirow[t]{3}{*}{ MS Classifier } & $k=2$ & 0.0827 & 3.2981 & -2.6064 & -3.7382 & -0.0094 & 0.3906 & -0.0210 \\
\hline & & & & & & -0.0122 & -0.0210 & 0.5386 \\
\hline & & & & & & 0.5373 & -0.0172 & -0.0039 \\
\hline \multirow[t]{3}{*}{ ASD Classifier } & $k=3$ & 0.1130 & 2.3453 & -0.3484 & 0.5773 & -0.0172 & 0.0608 & -0.0053 \\
\hline & & & & & & -0.0039 & -0.0053 & 0.1883 \\
\hline & & & & & & 0.1403 & 0.0107 & 0.0063 \\
\hline \multirow[t]{3}{*}{ NM Classifier } & $k=4$ & 0.1683 & 2.7874 & 1.8620 & -0.9829 & 0.0107 & 0.2549 & 0.0016 \\
\hline & & & & & & 0.0063 & 0.0016 & 0.1301 \\
\hline & & & & & & 0.0972 & 0.0077 & -0.0161 \\
\hline \multirow[t]{3}{*}{ AS Classifier } & $k=5$ & 0.0783 & 0.7511 & 0.3199 & -0.5341 & 0.0077 & 0.0344 & -0.0050 \\
\hline & & & & & & -0.0161 & -0.0050 & 0.2634 \\
\hline & & & & & & 0.3301 & -0.0011 & 0.0025 \\
\hline \multirow[t]{3}{*}{ AR Classifier } & $k=6$ & 0.2676 & -1.2294 & 0.1198 & 0.3222 & -0.0011 & 0.0230 & 0.0005 \\
\hline & & & & & & 0.0025 & 0.0005 & 0.3255 \\
\hline & & & & & & 0.1338 & 0.0048 & -0.0155 \\
\hline \multirow[t]{2}{*}{ VSD Classifier } & $k=7$ & 0.0954 & -0.1631 & -1.1167 & 0.9454 & 0.0048 & 0.1449 & -0.0095 \\
\hline & & & & & & -0.0155 & -0.0095 & 0.1573 \\
\hline
\end{tabular}
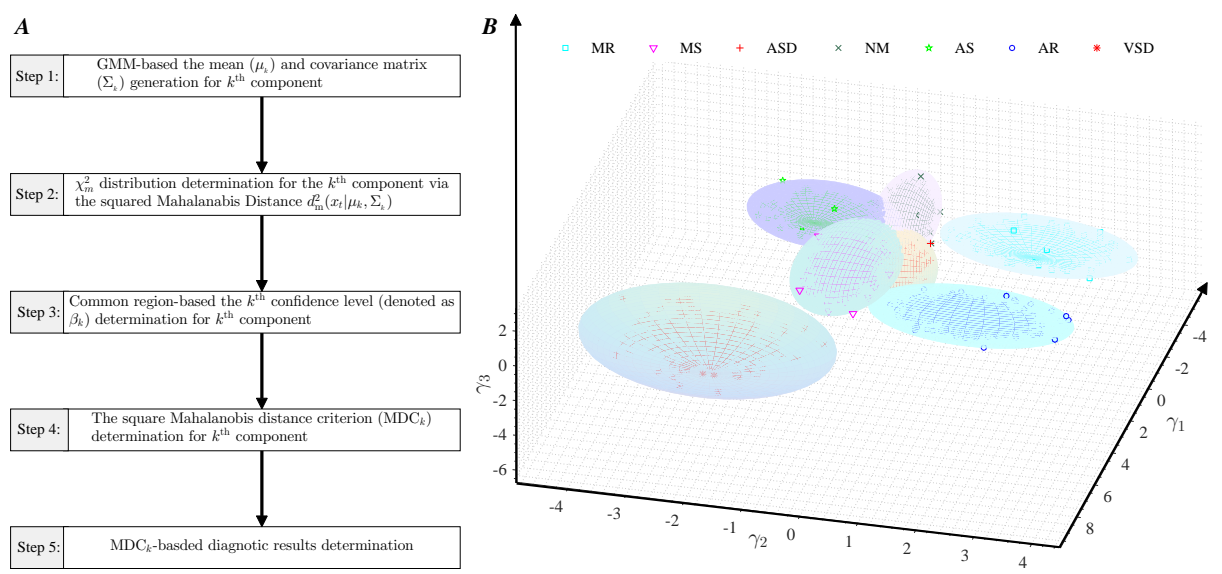

Fig. 8. Flow chart of the diagnostic determination and 3-dimensional surface classifier results. 
as possible, and the independent 3-dimensional interspace corresponding to each component is considered.

Step 2. $\chi_{3}^{2}$ determination for the $k^{\text {th }}$ component in 3-dimensional interspace Since the squared Mahalanobis distances for each Gaussian component follow the Chi-square distribution $\left(\chi_{3}^{2}\right)$ in 3-dimensional interspace, to determine the decision region for classifying the test data $\mathbf{x}$ via the components estimated in the above step, the squared Mahalanobis distance in 3-dimensional interspace for the $k^{\text {th }}$ component with mean $\mu_{k}$ and full covariance matrix $\Sigma_{k}, d_{3}^{2}\left(\mathbf{x} \mid \mu_{k}, \Sigma_{k}\right)$, is computed as follows:

$$
d_{3}^{2}\left(\mathbf{x} \mid \mu_{k}, \Sigma_{k}\right)=\left(\mathbf{x}-\mu_{k}\right)^{\mathrm{T}} \Sigma_{k}^{-1}\left(\mathbf{x}-\mu_{k}\right)
$$

Therefore, $\chi_{3}^{2}$, which is constructed based on component $k$ and denoted as $\chi_{3}^{2}\left(\mu_{k}, \Sigma_{k}\right)$, is determined by

$$
d_{3}^{2}\left(\mathbf{x} \mid \mu_{k}, \Sigma_{k}\right) \sim \chi_{3}^{2}\left(\mu_{k}, \Sigma_{k}\right)
$$

Therefore, the squared Mahalanobis distance $d_{3}^{2}\left(\mathbf{x} \mid \mu_{k}, \Sigma_{k}\right)$ specified based on the desired confidence level, denoted as $\beta_{k}$, can be used as the $k$ th classifier criterion for determining whether feature $\mathbf{x}$ belongs to the $k$ th class.

Step 3. The $k^{\text {th }}$ confidence level $\left(\beta_{k}\right)$ determination

Actually, the $k$ th confidence region, as specified by the $k$ th desired confidence level $\beta_{k}$, is surrounded by the $k$ th ellipsoid, and this relation is expressed as

$$
d_{3}^{2}\left(\mathbf{x} \mid \mu_{\mathbf{k}}, \boldsymbol{\Sigma}_{\mathbf{k}}\right) \leq \mathrm{MDC}_{\mathbf{k}}=\chi_{\mathbf{3}, \beta_{\mathbf{k}}}^{\mathbf{2}}
$$

where $\chi_{3, \beta_{k}}^{2}$ is the inverse of $\chi_{3}^{2}$ for a given confidence level $\beta_{k}$, and $\mathrm{MDC}_{k}$ represents the classification criterion for component $k$ and satisfies the following equation

$$
\chi^{2}\left(d_{3}^{2}\left(\mathbf{x} \mid \mu_{k}, \Sigma_{k}\right)=\mathrm{MDC}_{k} \mid \mu_{k}, \Sigma_{k}\right)=\beta_{k}
$$

For the $\chi_{3}^{2}$ distribution, although the confidence regions corresponding to the confidence levels of $68.3 \%, 95 \%$, and $97.5 \%$ are widely used classification criteria in many studies $[40,41,42,43,2]$, the optional $\beta_{k}$ is identified by setting $\beta_{k} \in[63: 2: 97] \%$ combined with the following rules: 1 ) each ellipsoid should be as large as possible; 2 ) each common region should be as small as possible; and 3) the classification accuracy defined in Eq. (22) should be as high as possible. The classification accuracies for classifying sound data summarized in subsection are plotted in Fig. 9, and Fig. 9 shows the following results.

F For VSD sounds, high accuracy can be achieved by setting the desired confidence level $\beta$ to each value within the interval of $(0.71<\beta<0.89)$, as shown in Fig. 9(VSD).

For AR and MR sounds, by setting the desired confidence level $\beta$ based on $\beta \in[0.69,0.81]$, high classification accuracy could be achieved (Fig. 9(MR and AR) ) ; 

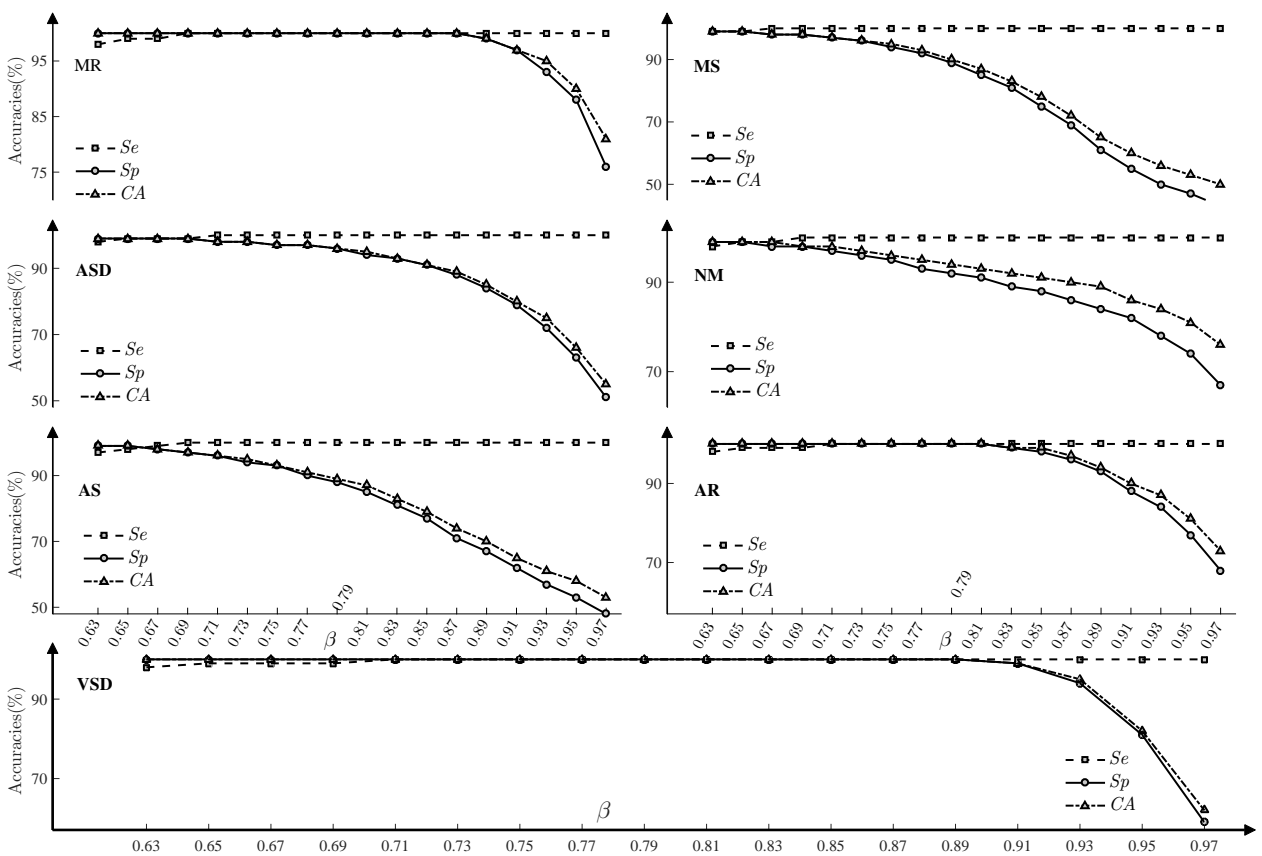

Fig. 9. The achieved accuracies corresponding to classifying the heart sounds described in subsection by setting $\beta$ form 0.63 to 0.97 with a step of 0.02 .

For MS, AS and NM sounds: To achieve the accurate classification of HSs, the interval of the desired confidence level $\beta$ should be set as [0.63,0.65] (Fig. 9).

For ASD sounds: Fig. 9(ASD) shows that the highest classification accuracy is achieved by setting the desired confidence level to $\beta \in[0.63,0.69]$.

Furthermore, the desired confidence level $\beta$ can be adjusted to improve the classification accuracy and fit new datasets without reperforming the computations for the objective function, especially for VSD sounds and MR sounds (Fig. 9(VSD and $\mathbf{M R}$ )). In this study, according to the rules described above combined with the accuracy analysis results plotted in Fig. 9 , the $\beta_{k}(k=1, \ldots, 7)$ values are set as $0.87,0.65,0.67,0.65,0.67,0.79$ and 0.87 , respectively.

Step 4. $\mathrm{MDC}_{k}$ determination corresponding to $\beta_{k}$

Based on the $k^{\text {th }}$ confidence level achieved for $\beta_{k}$ in the above step, by using the function 'chi2inv' in MATLAB 2018b, the inverse of $\chi_{3, \beta_{k}}^{2}$, denoted as $\mathrm{MDC}_{k}=$ $\chi_{3, \beta_{k}}^{2}$, is determined. The analysis results for the $k^{\text {th }}$ confidence region in the 3 dimensional interspace, which is surrounded by the $k^{\text {th }}$ ellipsoid corresponding to the $k^{\text {th }}$ desired confidence level $\beta_{k}$, are determined and shown in Fig. $8 \boldsymbol{B}$. Furthermore, Fig. $8 \boldsymbol{B}$ shows that the common regions between two random ellipsoids are almost zero; thus, a faulty decision process is avoided because the input will not fall into two or more categories.

Step 5. $\mathrm{MDC}_{\mathrm{k}}$-based diagnostic result determination

According to the confidence region shown in Fig. $8 \boldsymbol{B}, \mathrm{MDC}_{\mathrm{k}}$-based diagnostic results for a test feature $\mathbf{x}=\left[\gamma_{1}, \gamma_{2}, \gamma_{3}\right]$ are determined by

$\left\{\begin{array}{l}\text { Class } k, d_{3}^{2}\left(\mathbf{x} \mid \mu_{\mathbf{k}}, \boldsymbol{\Sigma}_{\mathbf{k}}\right) \leq \mathrm{MDC}_{\mathbf{k}} \\ \text { Unknown class, } \quad \text { otherwise }\end{array}\right.$, 
where class $k$ corresponding to the type of heart disease is detailed in Table 3 , and $\mathrm{MDC}_{k}(1,2, \ldots, 7)$ is 5.6489, 3.2831, 3.4297, 3.2831, 3.4297, 4.5258 and 5.6489. Moreover, to evaluate the performance of these ellipsoids in 3-dimensional space, the classification accuracy $(C A)$, sensitivity $(S e)$ and specificity $(S p)$ values are calculated by

$$
\left\{\begin{array}{l}
C A(\%)=\frac{\mathrm{TP}+\mathrm{TN}}{\mathrm{TP}+\mathrm{FP}+\mathrm{FN}+\mathrm{TN}} \times 100 \\
S e(\%)=\frac{\mathrm{TP}}{\mathrm{TP}+\mathrm{FN}} \times 100 \\
S p(\%)=\frac{\mathrm{TN}}{\mathrm{FP}+\mathrm{TN}} \times 100
\end{array}\right.
$$

where TP, FP, TN and FN are the numbers of true positives, false positives, true negatives and false negatives, respectively. To evaluate the performance of this diagnostic system, the classification method based on the ellipsoids shown in Fig. 8 is applied.

Definition of the diagnosis results

Based on the ellipsoid surfaces, the diagnosis method is described as follows.

(a) The 3-dimensional diagnostic features $\left[\gamma_{1}, \gamma_{2}, \gamma_{3}\right]$ are first transformed from the features FF (denoted as $\mathrm{FF}_{\mathrm{s}}$ ) of the testing sample and calculated with the following equation

$$
\gamma_{i}=\frac{\mathrm{FF}_{s}-\mu_{\mathrm{FF}}}{\sigma_{\mathrm{FF}}} \times \xi_{i}, i=1,2,3
$$

where $\mu_{\mathrm{FF}}$ and $\sigma_{\mathrm{FF}}$ are shown in Table 1.

(b) Then, according to the confidence region shown in Fig. $8 \boldsymbol{B}$, the $\mathrm{MDC}_{\mathrm{k}}$-based diagnostic result for a test features $\mathbf{x}=\left[\gamma_{1}, \gamma_{2}, \gamma_{3}\right]$ is determined by Eq. (22).

\section{System performance evaluation}

The performance of the proposed methodology is evaluated by comparison with the efficient methods summarized in Table 4. The comparison of the results of sound diagnosis is summarized in Table 5, and results are shown in Table 6 . The following results are given in Table 6 .

Method $\sharp 1$. The highest classification accuracies were obtained based on the rules described in a previous study [8].

* Method $\sharp 2$. The highest $C A$ results were obtained based on the following rules. Rule 1: If the $8^{\text {th }}$ value of Lyapunov exponent $\left(\mathrm{LPE}_{8}\right) \geq 0.79$ and $\left(\mathrm{LPE}_{9}\right) \leq 0.38$ then the heart is normal.

Rule 2: If $\mathrm{LPE}_{2} \leq 0.17$ and $\left(\mathrm{LPE}_{8}\right) \leq 0.79$, then the heart disease is VSD.

Rule 3: If $\mathrm{LPE}_{4} \geq 0.17, \mathrm{LPE}_{6} \leq 0.39$, and $\mathrm{LPE}_{3} \leq 0.56$, then the heart disease is MR.

Rule 4: If $\mathrm{LPE}_{5} \geq 0.17, \mathrm{LPE}_{4} \geq 0.67$, and $\mathrm{LPE}_{3} \geq 0.37$, then the heart disease is MS.

Rule 5: If $\mathrm{LPE}_{7} \geq 0.54, \mathrm{LPE}_{3} \geq 0.29$, and $\mathrm{LPE}_{5} \geq 0.49$, then the heart disease is AR.

Rule 6: If $\mathrm{LPE}_{8} \geq 0.39, \mathrm{LPE}_{5} \geq 0.72$, and $\mathrm{LPE}_{2} \leq 0.68$, then the heart disease is ASD. 
Table 4.

Efficient methods successfully used in diagnosing normal sounds from other common heart diseases

\begin{tabular}{|c|c|c|}
\hline Method & Refs & Performance evaluation \\
\hline$\sharp 1$ & [8] & $\begin{array}{l}\text { The higher CA, achieved in this study, was } 95.5 \%, 92.1 \%, 96.2 \% \text { and } 99.0 \% \text { for diagnosing small } \\
\text { ventricular septal defect (VSD), moderate VSD, large VSD and normal sounds, respectively. }\end{array}$ \\
\hline$\sharp 2$ & {$[44]$} & $\begin{array}{l}\text { A rule-based classification tree method proposed by this study achieved very high CA: } 95.45 \% \text { for } \\
\text { diagnosing VSD, } 100 \% \text { for diagnosing normal, } 100 \% \text { for diagnosing aortic stenosis and } 95.45 \% \text { for } \\
\text { diagnosing aortic insufficiency. }\end{array}$ \\
\hline$\sharp 3$ & {$[45]$} & $\begin{array}{l}\text { Artificial neural networks (ANNs) was reported to achieve the second-best score compared to the } \\
\text { other methods in classifying the phonocardiogram recordings provided by the CinC Challenge. }\end{array}$ \\
\hline$\sharp 4$ & {$[46]$} & $\begin{array}{l}\text { Random forest, a meta-learning approach that uses multiple random decision trees as base learners and } \\
\text { aggregates them to compute the final ensemble prediction, was successfully used in sound classification } \\
\text { such as studies. }\end{array}$ \\
\hline$\sharp 5$ & {$[47]$} & $\begin{array}{l}\text { Tunable- } Q \text { wavelet transform approach proposed by this study, which yielded more successful diag- } \\
\text { nosing results for distinguishing septal defect from NM with an accuracy of } 100 \% \text { at the tenth level } \\
\text { of decomposition for } Q=6 \text {. }\end{array}$ \\
\hline$\sharp 6$ & {$[48]$} & $\begin{array}{l}\text { the multi-level basis selection (MLBS) method proposed in this study, which achieved a higher speci- } \\
\text { ficity of } 98.31 \% \text { for diagnosing MR than other selection methods }\end{array}$ \\
\hline$\sharp 7$ & [49] & $\begin{array}{l}\text { The PCA-DHMM approach proposed in this study yielded more successful diagnosing results for } \\
\text { detecting PS with an accuracy of } 98.3 \% \text { than other studies }[50,51]\end{array}$ \\
\hline$\sharp 8$ & {$[11]$} & $\begin{array}{l}\text { The multi-support vector machine (M-SVM) method achieved very high classification accuracies: } \\
96.88 \% \text { for diagnosing AF, } 93.94 \% \text { for diagnosing MR, and } 99.8 \% \text { for diagnosing NM. }\end{array}$ \\
\hline
\end{tabular}

Table 5. Experimental sounds used to evaluate the performance

\begin{tabular}{lccccccr}
\hline \multirow{2}{*}{ Data source } & \multicolumn{6}{c}{ Period numbers of every type of heart disease/Patients } \\
\cline { 2 - 8 } & MR & MS & ASD & NM & AS & AR & VSD \\
\hline sounds in subsection & $769 / 10$ & $439 / 5$ & $315 / 7$ & $1056 / 45$ & $381 / 10$ & $665 / 15$ & $327 / 10$ \\
New sounds & $156 / 3$ & $132 / 2$ & $82 / 2$ & $183 / 8$ & $126 / 3$ & $153 / 4$ & $70 / 3$ \\
Total sounds & $925 / 13$ & $571 / 7$ & $397 / 9$ & $1239 / 53$ & $507 / 13$ & $818 / 19$ & $397 / 13$ \\
\hline
\end{tabular}

Rule 7: If $\mathrm{LPE}_{9} \geq 0.64, \mathrm{LPE}_{3} \geq 0.39$, and $\mathrm{LPE}_{7} \geq 0.21$, then the heart disease is AS.

Rule 8: If none of these conditions are met, the $\mathbf{H S}$ is undefined.

Method $\sharp 3$. The most accurate results were obtained by the structure consisting of one input layer with 60 neurons, one hidden layer with 11 neurons and one output layer with five neurons.

Method $\sharp 4$. The most accurate results were obtained by setting the number of features at each node, the number of trees and the maximum depth of trees to 1 , 108 , and 36, respectively.

Method $\sharp 5$. The most accurate results were obtained by setting $Q=3,2,5,6,2,5$ and 3 .

W Method $\sharp 6$. The most accurate resutls were obtained by setting the sample parameters to the values given in [10].

Method $\sharp 7$. The highest $C A$ was obtained by using the Euclidean metric in the distance calculation for the $\mathbf{K N N}$ classifier.

Method $\sharp 8$. The accuracy of the results obtained with the PCA-DHMM method was the highest for the detection of MR, MS, ASD, NM, AS, AR and VSD by setting the $p$ parameters to $3,5,8,3,7,8$ and 8 respectively.

Method $\sharp 9$. The most accurate results were obtained for the diagnosis of $\mathbf{M R}$, MS, ASD, NM, AS, AR and VSD at $T h v=0.4,0.3,0.2,0.2,0.4,0.1$ and 0.2 , respectively.

The results in Table 6 support the following conclusions. 
Table 6. Comparative analysis of eight different methods for the diagnosis of heart diseases summarized in Table 5

\begin{tabular}{|c|c|c|c|c|c|c|c|c|c|c|c|c|c|c|c|c|c|c|c|c|c|}
\hline \multirow{2}{*}{ Method - } & \multirow{2}{*}{\multicolumn{3}{|c|}{ MR }} & \multicolumn{3}{|c|}{$\begin{array}{c}\mathrm{MS} \\
\end{array}$} & \multicolumn{3}{|c|}{$\begin{array}{l}\text { ASD } \\
\end{array}$} & \multicolumn{3}{|c|}{$\mathrm{NM}$} & \multicolumn{3}{|c|}{$\mathrm{AS}$} & \multicolumn{3}{|c|}{$\mathrm{AR}$} & \multicolumn{3}{|c|}{ VSD } \\
\hline & & & $\frac{S P(\%)}{S(\%)}$ & Se\% & $C A(\%)$ & $\frac{p(\%)}{p(\%)}$ & Se & $C A(\%)$ & p(\%) & $\mathrm{Se} \%$ & $A(\%)$ & $\overline{p p(\%)}$ & $5 e \%$ & $\frac{C A(\%)}{C A(6)}$ & $\frac{S p(\%)}{p p(\%)}$ & & $C A(\%)$ & $S p(\%)$ & & & $\begin{array}{ll}S p(\%) \\
p(\%)\end{array}$ \\
\hline$\$ 1$ & 90.1 & 87.34 & 86.7 & 83.2 & 86.81 & 87.3 & 90.2 & 83.13 & 82.5 & 85.7 & 81.90 & 80.6 & 91.3 & 90.40 & 90.3 & 87.2 & 84.04 & 83.4 & 96.2 & 97.66 & 97.8 \\
\hline$=2$ & 88.6 & 85.93 & 85.3 & 86.1 & 89.72 & 90.2 & 79.8 & 82.10 & 82.3 & 96.1 & 98.93 & & 98.3 & 91.95 & 96.2 & 85.1 & 86.97 & 83.6 & 87.5 & 87.19 & 82.1 \\
\hline$\sharp 3$ & 88.1 & 87.6 & 87.8 & 83.1 & 89.36 & 90.2 & 80.3 & 81.67 & 81.8 & 92.6 & 91.63 & 91.3 & 83.9 & 86.04 & 86.3 & 90.1 & 84.69 & 83.6 & 87.6 & 86.03 & .9 \\
\hline 4 & 89.7 & 91.64 & 92.1 & 85.2 & 83.52 & 83.3 & 86.3 & 87.49 & 87.6 & 93.7 & 91.61 & 90.9 & 90.1 & 87.05 & 86.7 & 85.2 & 82.21 & 81.6 & 90.8 & 91.63 & .7 \\
\hline 45 & 87.9 & 91.29 & 92.1 & 87.9 & 90.72 & 91.1 & 93.2 & 87.59 & 87.1 & 9 & 95.36 & 96.9 & 90.6 & 88.54 & 88.3 & 83.1 & 82.43 & 82.3 & 91.7 & 90.65 & 19 \\
\hline 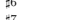 & 93.6 & ${ }^{25} \cdot 30$ & ${ }^{95 . .1}$ & 88.1 & 83.78 & 83.2 & 83.6 & 84.19 & 84.9 & 2 & $80 . \pi / 7$ & 85.6 & 3 & 80.31 & 86.2 & 8.2 & 82.29 & 81.3 & 89.3 & 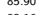 & 1.6 \\
\hline$=1$ & 11.0. & 87.03 & o.t & 85.2 & . & . & 8. & 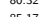 & & 定. & 13. & 舅. & 勇. & (83.23 & (8.1. & (9.3.5. & 染 & 80.9 & & 10 & 11 \\
\hline This & . & 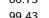 & 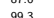 & 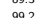 & 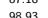 & 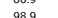 & 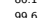 & 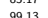 & 然 & 100 & 资, & 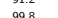 & 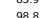 & 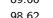 & 8.16 & 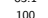 & 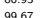 & 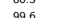 & 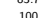 & 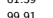 & 2 \\
\hline
\end{tabular}

(a) Although using method \#2 to diagnose NM yielded a higher $S p$ than that of the proposed method, the $C A$ was lower than that of the proposed method, partially due to the high $S e$ achieved by the proposed method.

(b) For other sounds, the classification accuracies achieved the proposed method were all greater than those of the other methods listed in Table 6 .

Overall, the efficiency of the proposed method in diagnosing MR, MS, ASD, NM, AS, AR and VSD diseases was evaluated by comparison with the other efficient methods listed in Table 6 .

\section{Conclusion}

A novel intelligent system was proposed for diagnosing heart diseases with high $C A$. The innovation of this approach is primarily reflected in: 1) the automatic extraction of secondary envelope-based frequency features; 2) the automatic determination of PCA-based diagnostic features $\gamma_{1}, \gamma_{2}$ and $\gamma_{3}$; and 3) the determination of adjustable confidence regions corresponding to the $\chi^{2}$ distribution. The confidence regions are obtained by calculating the Mahalanobis distance, which is adjusted by the desired confidence level $\beta$, and the results are used as the classification criteria for diagnosing heart diseases. The procedure for the implementation of the intelligent system involves three stages. Stage 1 describes the location and extraction of STMHT-based $C S_{1}$ and $C S_{2}$. In stage 2, in the frequency domain, a novel method was first proposed to generate the envelopes $C S_{1 \mathrm{~F}_{\mathrm{E}}}$ and $C S_{2 \mathrm{~F}_{\mathrm{E}}}$; then, based on the Thv lines, FF is automatically extracted. Finally, based on $\mathbf{P}$ CA, the first three principal components, $\gamma_{1}, \gamma_{2}$ and $\gamma_{3}$, which express $86.7 \%$ of the FF information, were determined and used as diagnostic features. In stage 3 , the GMM-based objective function $f_{\text {et }}(\mathbf{x})$ with respect to the features $\mathbf{x}=\left[\gamma_{1}, \gamma_{2}, \gamma_{3}\right]$ and the parameters $\left[\pi_{k}, \mu_{k}, \Sigma_{k}\right]$, where $k=1,2, \ldots, K$, was generated. Then, the $\chi^{2}$ distribution for component $k$ was determined by calculating the Mahalanobis distance from $\mathbf{x}$ to the class mean $\mu_{k}$ of component $k$, and the confidence region for component $k$ was determined by adjusting the optimal confidence level $\beta_{k}$ and used as the criterion (denoted as $\mathrm{MDC}_{k}$ ) to diagnose a given HS. The performance evaluation was validated by sounds from online $\mathbf{H S}$ databases and clinical heart databases. The accuracy of the proposed method was compared to the accuracies of other well-known classifiers, and the highest classification accuracies of $99.43 \%$, $98.93 \%, 99.13 \%, 99.85 \%, 98.62 \%, 99.67 \%$ and $99.91 \%$ in the detection of MR, MS, ASD, NM, AS, AR and VSD sounds were achieved by setting $\beta_{k}(k=1,2, \ldots, 7)$ to $0.87,0.65,0.67,0.65,0.67,0.79$ and 0.87 , respectively. Therefore, this proposed intelligent diagnosis system provides an efficient way to diagnose seven types of heart diseases. In addition, methods to manage the sounds (such as some ASD sounds) 
when $C S_{1}$ and $C S_{2}$ cannot be segmented and extracted via the STMHT method will be explored in the future to characterize the physical meanings of the frequency components and to build a model of the secondary curve in the frequency domain.

\section{Acknowledgements \\ Not applicable}

Funding

Not applicable

\section{Abbreviations}

HSs: Heart sounds; $S_{1}$ : First heart sound; $S_{2}$; Second heart sound; STMHT: Short time modified Hilbert transform; $\left(C S_{1}\right)$ : First complex sound; $\left(C S_{2}\right)$ : Second complex sound; $C S_{1 \mathrm{~F}_{\mathrm{E}}}$ : Frequency envelope corresponding to $\left(C S_{1}\right) ; C S_{2 \mathrm{~F}_{\mathrm{E}}}$ : Frequency envelope corresponding to $\left(C S_{2}\right)$; Thv: Threshold value; PCA: Principal component analysis; $\gamma_{1}$ : First principal component; $\gamma_{2}$ : Second principal component; $\gamma_{3}$ : Third principal component; GMM: Gaussian mixture model; $f_{e t}(\mathbf{x})$ : Objective function; $\chi^{2}$ : Chi-squared distribution; $\mu_{k}$ the $k^{t h}$ class mean; $\beta_{k}: k^{\text {th }}$ confidence level;

\section{Availability of data and materials} Not applicable

Ethics approval and consent to participate

This study protocol was approved by the local ethics committee of Nanyang Institute of Technology and the First peoples hospital of Nanyang, China. We followed data collection of WHO guidance on heart sound auscultation to perform this study.

\section{Competing interests}

The authors declare that they have no competing interests.

Consent for publication

Not applicable

\section{Authors' contributions}

Sun,Biqiang Zhang, He: collection, organizing, and review of the literature. Sun, Biqing Zhang and Huang: preparing the manuscript. Sun, Biqiang Zhang, Fan, Yan, Jiale Zhang and Chen: manuscript revision and modification. All authors read and approved the final manuscript.

Authors' information

${ }^{1}$ Department of Information Engineering, Nanyang Institute of Technology, 473004, Nanyang city, China. ${ }^{2}$ College of Physics and Optoelectronic Engineering, Shenzhen University, 518061, Shenzhen, China

\section{Author details}

${ }^{1}$ Department of Information Engineering, Nanyang Institute of Technology, Nanyang, China. ${ }^{2}$ College of Physics and Optoelectronic Engineering, Shenzhen University, Shenzhen, China.

\section{References}

1. Tan, Z., Wang, W., Zong, R., Pan, J., Yang, H.: [classification of heart sound signals in congenital heart disease based on convolutional neural network]. Sheng wu yi xue gong cheng xue za zhi = Journal of biomedical engineering $=$ Shengwu yixue gongchengxue zazhi 36(5), 728-736 (2019). doi:10.7507/1001-5515.201806031

2. Sun, S., Wang, H., Chang, Z., Mao, B., Liu, Y.: On the Mahalanobis Distance Classification Criterion for a Ventricular Septal Defect Diagnosis System. IEEE Sensors Journal 19(7), 2665-2674 (2019). doi:10.1109/JSEN.2018.2882582

3. Omari, T., Bereksi-Reguig, F.: A new approach for blood pressure estimation based on phonocardiogram. Biomedical Engineering Letters 9(3), 395-406 (2019). doi:10.1007/s13534-019-00113-z

4. Notario, P.M., Gentile, E., Amidon, M., Angst, D., Lefaiver, C., Webster, K.: Home-based telemedicine for children with medical complexity. Telemedicine and e-Health (2019)

5. Coviello, J.S.: Auscultation Skills: Breath \& Heart Sounds. -5th Ed., 5th edn., p. 256. Lippincott Williams \& Wilkins, ??? (2013). doi:10.1136 bmj.320.7229.256

6. Liu, Q., Wu, X., Ma, X.: An automatic segmentation method for heart sounds. BioMedical Engineering Online 17(1), 1-22 (2018). doi:10.1186/s12938-018-0538-9

7. Messner, E., Zöhrer, M., Pernkopf, F.: Heart sound segmentation - An event detection approach using deep recurrent neural networks. IEEE Transactions on Biomedical Engineering 65(9), 1964-1974 (2018). doi:10.1109/TBME.2018.2843258

8. Sun, S., Wang, H.: Principal component analysis-based features generation combined with ellipse models-based classification criterion for a ventricular septal defect diagnosis system. Australasian Physical \& Engineering Sciences in Medicine (2018). doi:10.1007/s13246-018-0676-1

9. Sun, S.: An innovative intelligent system based on automatic diagnostic feature extraction for diagnosing heart diseases. Knowledge-Based Systems 75, 224-238 (2015). doi:10.1016/j.knosys.2014.12.001

10. Shuping Sun, Z.J.Y.F. Haibin Wang, Tao, T.: Segmentation-based heart sound feature extraction combined with classifier models for a \{VSD $\}$ diagnosis system. Expert Systems with Applications 41(4, Part 2), 1769-1780 (2014). doi:10.1016/j.eswa.2013.08.076 
11. Choi, S., Jiang, Z.: Cardiac sound murmurs classification with autoregressive spectral analysis and multi-support vector machine technique. Computers in Biology and Medicine 40(1), 8-20 (2010). doi:10.1016/j.compbiomed.2009.10.003

12. Zhang, J., Yin, Z., Wang, R.: Pattern Classification of Instantaneous Cognitive Task-load Through GMM Clustering, Laplacian Eigenmap, and Ensemble SVMs. IEEE/ACM Transactions on Computational Biology and Bioinformatics 14(4), 947-965 (2017). doi:10.1109/TCBB.2016.2561927

13. Li, Z., Xia, Y., Ji, Z., Zhang, Y.: Brain voxel classification in magnetic resonance images using niche differential evolution based Bayesian inference of variational mixture of Gaussians. Neurocomputing 269, 47-57 (2017). doi:10.1016/j.neucom.2016.08.147

14. Ortiz-Rosario, A., Adeli, H., Buford, J.A.: MUSIC-Expected maximization gaussian mixture methodology for clustering and detection of task-related neuronal firing rates. Behavioural Brain Research 317, 226-236 (2017) doi:10.1016/j.bbr.2016.09.022

15. Davari, A., Aptoula, E., Yanikoglu, B., Maier, A., Riess, C.: GMM-Based Synthetic Samples for Classification of Hyperspectral Images With Limited Training Data. IEEE Geoscience and Remote Sensing Letters 15(6), 942-946 (2018). doi:10.1109/LGRS.2018.2817361. 1712.04778

16. Simms, L.M., Blair, B., Ruz, J., Wurtz, R., Kaplan, A.D., Glenn, A.: Nuclear Inst . and Methods in Physics Research , A Pulse discrimination with a Gaussian mixture model on an FPGA. Nuclear Inst. and Methods in Physics Research, A 900(May), 1-7 (2018). doi:10.1016/j.nima.2018.05.039

17. Xue, W., Jiang, T.: An adaptive algorithm for target recognition using Gaussian mixture models. Measurement: Journal of the International Measurement Confederation 124(10), 233-240 (2018). doi:10.1016/j.measurement.2018.04.019

18. Zhang, S., Chen, X., Zhu, Z., Feng, B., Chen, Y., Long, W.: Segmentation of small ground glass opacity pulmonary nodules based on Markov random field energy and Bayesian probability difference. BioMedical Engineering Online 19(1), 1-20 (2020). doi:10.1186/s12938-020-00793-0

19. Ali, M.N., El-Dahshan, E.-S.A., Yahia, A.H.: Denoising of Heart Sound Signals Using Discrete Wavelet Transform. Circuits, Systems, and Signal Processing 36(11), 4482-4497 (2017). doi:10.1007/s00034-017-0524-7

20. Máttar, J.A., Shoemaker, W.C., Diament, D., Lomar, A., Lopes, A.C., De Freitas, E., Stella, F.P., Factore, L.A.: Systolic and diastolic time intervals in the critically ill patient. Critical care medicine 19(11), 1382-6 (1991)

21. Yeo, T.C., Dujardin, K.S., Tei, C., Mahoney, D.W., McGoon, M.D., Seward, J.B.: Value of a doppler-derived index combining systolic and diastolic time intervals in predicting outcome in primary pulmonary hypertension. The American Journal of Cardiology 81(9), 1157-1161 (1998). doi:10.1016/S0002-9149(98)00140-4

22. Cui, W., Roberson, D.A., Chen, Z., Madronero, L.F., Cuneo, B.F.: Systolic and diastolic time intervals measured from doppler tissue imaging: Normal values and z-score tables, and effects of age, heart rate, and body surface area. Journal of the American Society of Echocardiography 21(4), 361-370 (2008). doi:10.1016/j.echo.2007.05.034

23. Implementation, C.M.: Heart sounds databases-Continuing Medical Implementation. http://www. cvtoolbox . com/index.html (2019). http://www.cvtoolbox.com/index.html Accessed 11/12/19

24. MacWalter, D., MacWalter, G.: Human Heart Sounds and Murmurs. http://www.dundee.ac.uk/medther/ Cardiology/hsmur.html (2019). http://www.dundee.ac.uk/medther/Cardiology/hsmur.html

25. Shuping Sun, H.W.: A novel method-based secondary envelope extraction for heart sound analysis (2020)

26. 3MDatabase: 50 Heart and Lung Sounds Library. http://solutions.3m.com/wps/portal/3M/en_EU/ 3M-Littmann-EMEA/stethoscope/littmann-learning-institute/heart-lung-sounds/ heart-lung-sound-library/ (2019). http://solutions.3m.com/wps/portal/3M/en_EU/ 3M-Littmann-EMEA/stethoscope/littmann-learning-institute/heart-lung-sounds/ heart-lung-sound-library/

27. Medical Sound Library: Auscultate - Learn Heart Sounds, Murmurs and Medical Auscultation (2019). https:// www. medzcool. com/auscultate Accessed 2019-11-23

28. AMBOSSMed: AMBOSS: Medical Knowledge Distilled (2019). https://www.medzcool.com/auscultate Accessed 2019-09-25

29. Auscultation Sound: Heart Murmur-Mitral Regurgitation Auscultation Sound !!! Complete (2019). https:// www. medzcool.com/auscultate Accessed 2019-04-19

30. Sound, M.H., Library, M.: University of Michigan Heart Sound and Murmur Library. http://www. med.umich. edu/lrc/psb/heartsounds/index.htm (2019). http://www . med.umich.edu/lrc/psb/heartsounds/index. htm

31. ThinkLabs: Thinklabs heart sound library. https://www.thinklabs.com/heart-sounds?hc_location=ufi (2019). https://www.thinklabs. com/heart-sounds?hc_location=ufi Accessed 23/11/19

32. Mohseni, S.S.: Heart Arrhythmias Classification via a Sequential Classifier Using Neural Network, Principal Component Analysis and Heart Rate Variation. IEEE 8th International Conference on Intelligent Systems Heart, 715-722 (2016)

33. Kavitha, R., Kannan, E.: An efficient framework for heart disease classification using feature extraction and feature selection technique in data mining. 1st International Conference on Emerging Trends in Engineering, Technology and Science, ICETETS 2016 - Proceedings (2016). doi:10.1109/ICETETS.2016.7603000

34. Guo, H.-W., Huang, Y.-S., Lin, C.-H., Chien, J.-C., Haraikawa, K., Shieh, J.-S.: Heart Rate Variability Signal Features for Emotion Recognition by Using Principal Component Analysis and Support Vectors Machine. 2016 IEEE 16th International Conference on Bioinformatics and Bioengineering (BIBE), 274-277 (2016) doi:10.1109/BIBE.2016.40

35. Motin, M.A.: Principal Component Analysis : A Novel Approach for Extracting Respiratory Rate and Heart Rate From Photoplethysmographic Signal 22(3), 766-774 (2018)

36. H. El-Saadawy, H.A.S. M. Tantawi, Tolba, M.F.: Electrocardiogram (ecg) heart disease diagnosis using pnn, svm and softmax regression classifiers. The Eighth International Conference on Intelligent Computing and Information Systems (ICICIS) (ICICIS), 106-110 (2017) 
37. Rana, K.D., Rana, K.D., Khan, S., Khan, S., Khan, S., Hämäläinen, M.S., Hämäläinen, M.S., Vaina, L.M., Vaina, L.M., Vaina, L.M., Vaina, L.M.: A computational paradigm for real-time MEG neurofeedback for dynamic allocation of spatial attention. BioMedical Engineering Online 19(1), 1-17 (2020). doi:10.1186/s12938-020-00787-y

38. Johnson, R.A., Wichern, D.W.: Applied Multivariate Statistical Analysis (6th Edition), p. 800. Pearson, ??? (2007). http://www .amazon. com/Applied-Multivariate-Statistical-Analysis-Edition/dp/0131877151

39. Dempster, A.P., Laird, N.M., Rubin, D.B.: Maximum likelihood from incomplete data via the em algorithm. Journal of the Royal Statistical Society. Series B (Methodological) 39(1), 1-38 (1977)

40. Pinto, R.C., Engel, P.M.: A fast incremental gaussian mixture model. PLOS ONE 10(10), 1-12 (2015). doi:10.1371/journal.pone.0139931

41. Proïa, F., Pernet, A., Thouroude, T., Michel, G., Clotault, J.: On the characterization of flowering curves using Gaussian mixture models. Journal of Theoretical Biology 402, 75-88 (2016). doi:10.1016/j.jtbi.2016.04.022

42. Mungai, P.K.: Using Keystroke Dynamics in a Multi-level Architecture to Protect Online Examinations from Impersonation. 2017 IEEE 2nd International Conference on Big Data Analysis, 622-627 (2017)

43. Aryafar, A., Mikaeil, R., Doulati Ardejani, F., Shaffiee Haghshenas, S., Jafarpour, A.: Application of non-linear regression and soft computing techniques for modeling process of pollutant adsorption from industrial wastewaters. Journal of Mining and Environment (2018)

44. Karar, M.E., El-Khafif, S.H., El-Brawany, M.A.: Automated diagnosis of heart sounds using rule-based classification tree. Journal of Medical Systems 41(4), 60 (2017). doi:10.1007/s10916-017-0704-9

45. Zabihi, M., Bahrami Rad, A., Kiranyaz, S., Gabbouj, M., K. Katsaggelos, A.: Heart Sound Anomaly and Quality Detection using Ensemble of Neural Networks without Segmentation. Computing in Cardiology Conference (CinC), 2016 (2016). doi:10.22489/CinC.2016.180-213

46. Witten, I.H., Frank, E., Hall, M.: Data Mining: Practical Machine Learning Tools and Techniques, 4th edn., p 664. Morgan Kaufmann, ??? (2016). doi:0120884070, 9780120884070. arXiv:1011.1669v3. http://books . google. com/books?id=bDtLM8CODsQC\&pgis=1

47. Patidar, S., Pachori, R.B., Garg, N.: Automatic diagnosis of septal defects based on tunable-Q wavelet transform of cardiac sound signals. Expert Systems with Applications 42(7), 3315-3326 (2015). doi:10.1016/j.eswa.2014.11.046

48. Safara, F., Doraisamy, S., Azman, A., Jantan, A., Abdullah Ramaiah, A.R.: Multi-level basis selection of wavelet packet decomposition tree for heart sound classification. Computers in biology and medicine 43(10), 1407-14 (2013). doi:10.1016/j.compbiomed.2013.06.016

49. Saracoglu, R.: Hidden markov model-based classification of heart valve disease with pca for dimension reduction. Engineering Applications of Artificial Intelligence 25(7), 1523-1528 (2012). doi:10.1016/j.engappai.2012.07.005

50. Reed, T.R., Reed, N.E., Fritzson, P.: Heart sound analysis for symptom detection and computer-aided diagnosis. Simulation Modelling Practice and Theory 12, 129-146 (2004). doi:10.1016/j.simpat.2003.11.005

51. Sinha, R., Aggarwal, Y., Das, B.: Backpropagation artificial neural network classifier to detect changes in heart sound due to mitral valve regurgitation. Journal of Medical Systems 31(3), 205-209 (2007). doi:10.1007/s10916-007-9056-1 
Figures

Heart Sound (HS)

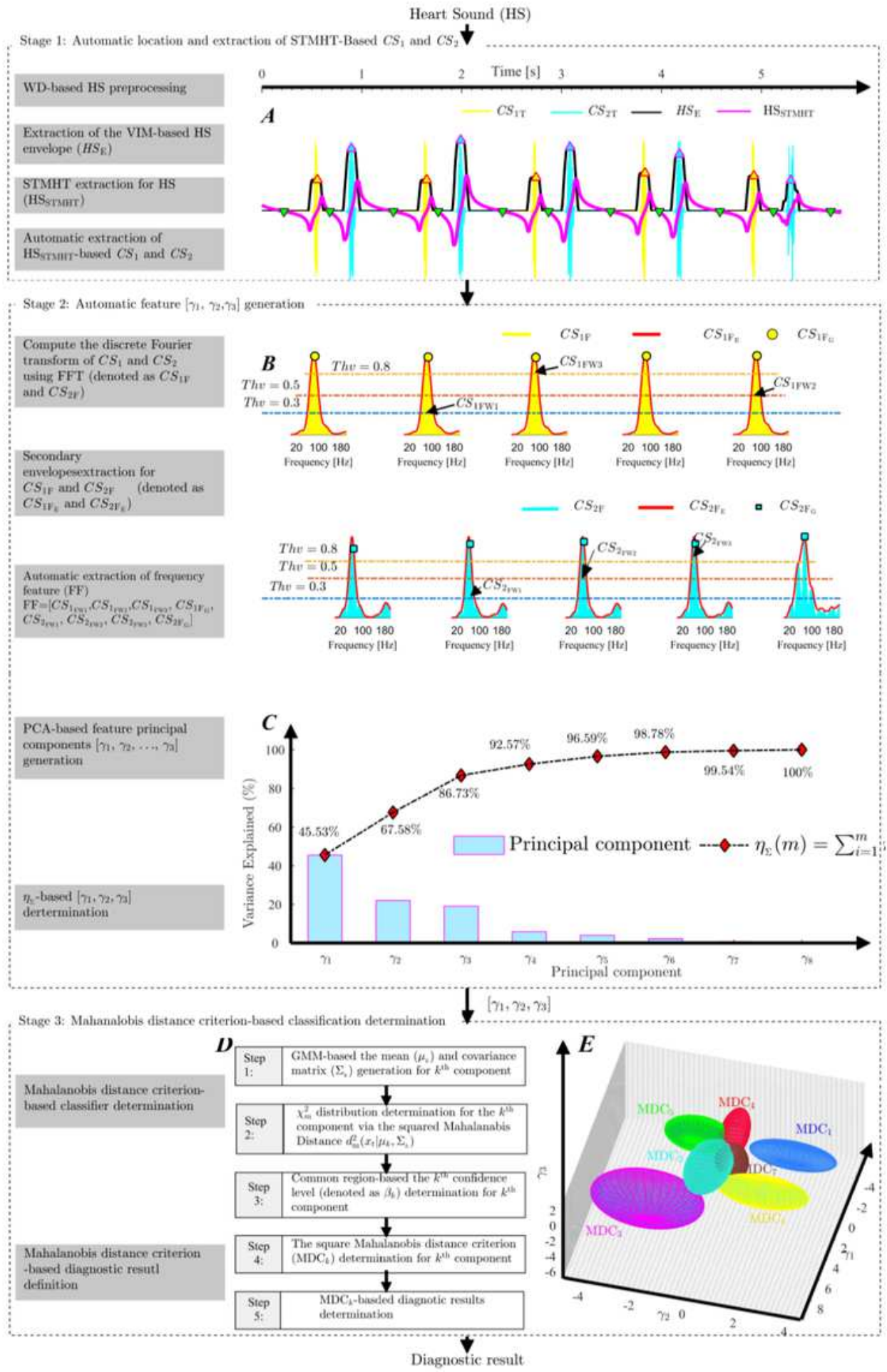

Figure 1

Flow chart of the proposed methodology. 


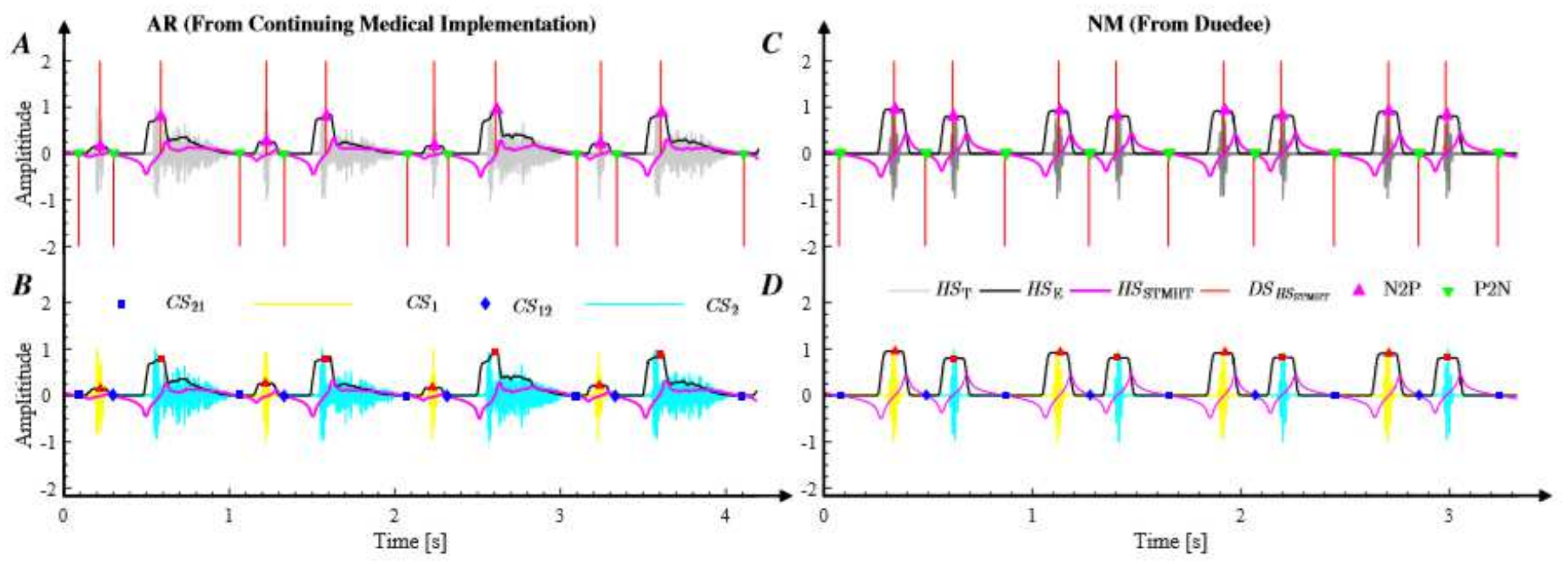

Figure 2

The automatic extraction procedures for CS1 and CS2. A-B show the procedure for an example of a typical AR from the database in [23]. C-D show the procedure for an example of a typical normal sound from [24].
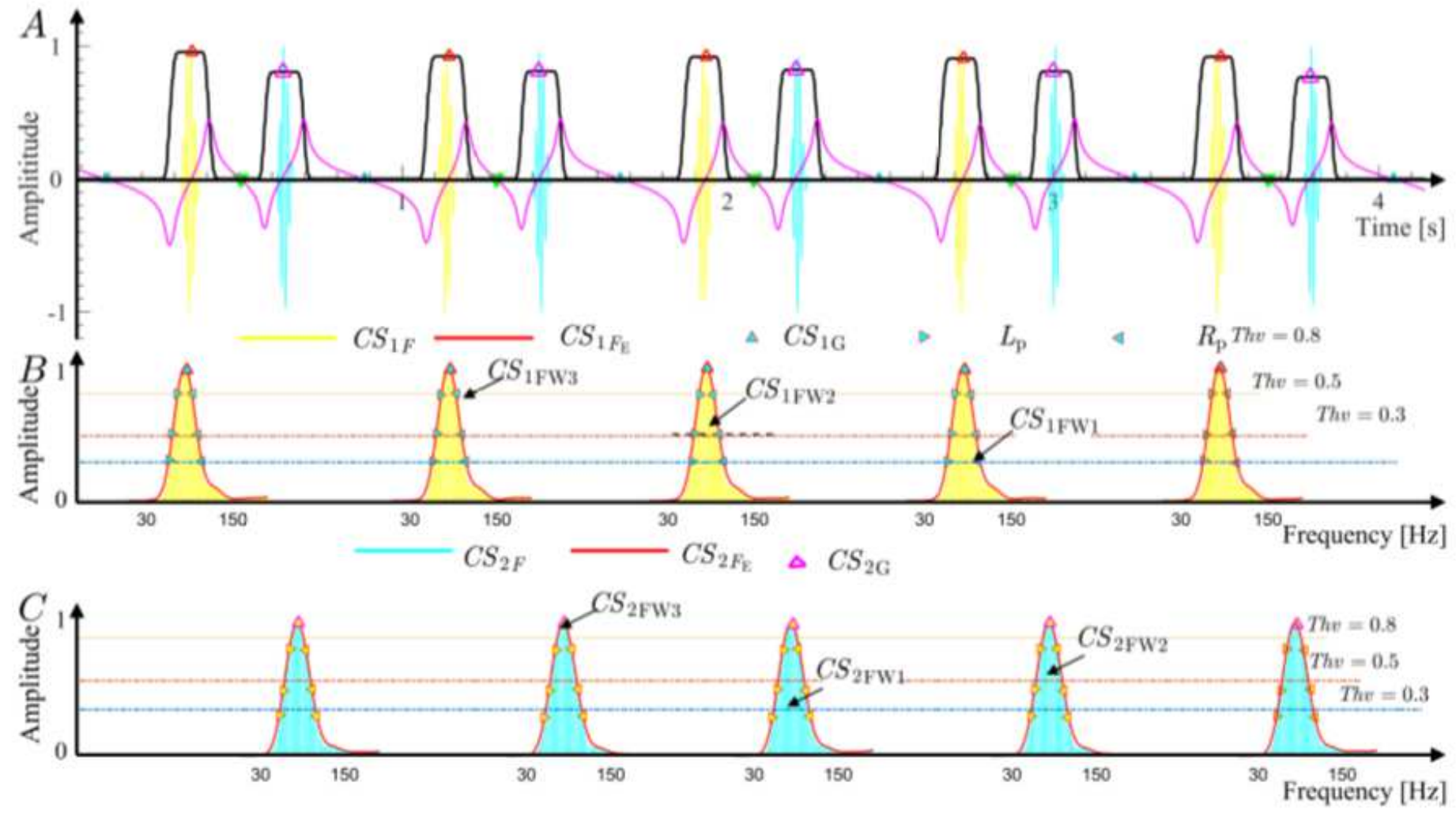

Figure 3

Example of feature definition and automatic extraction. 

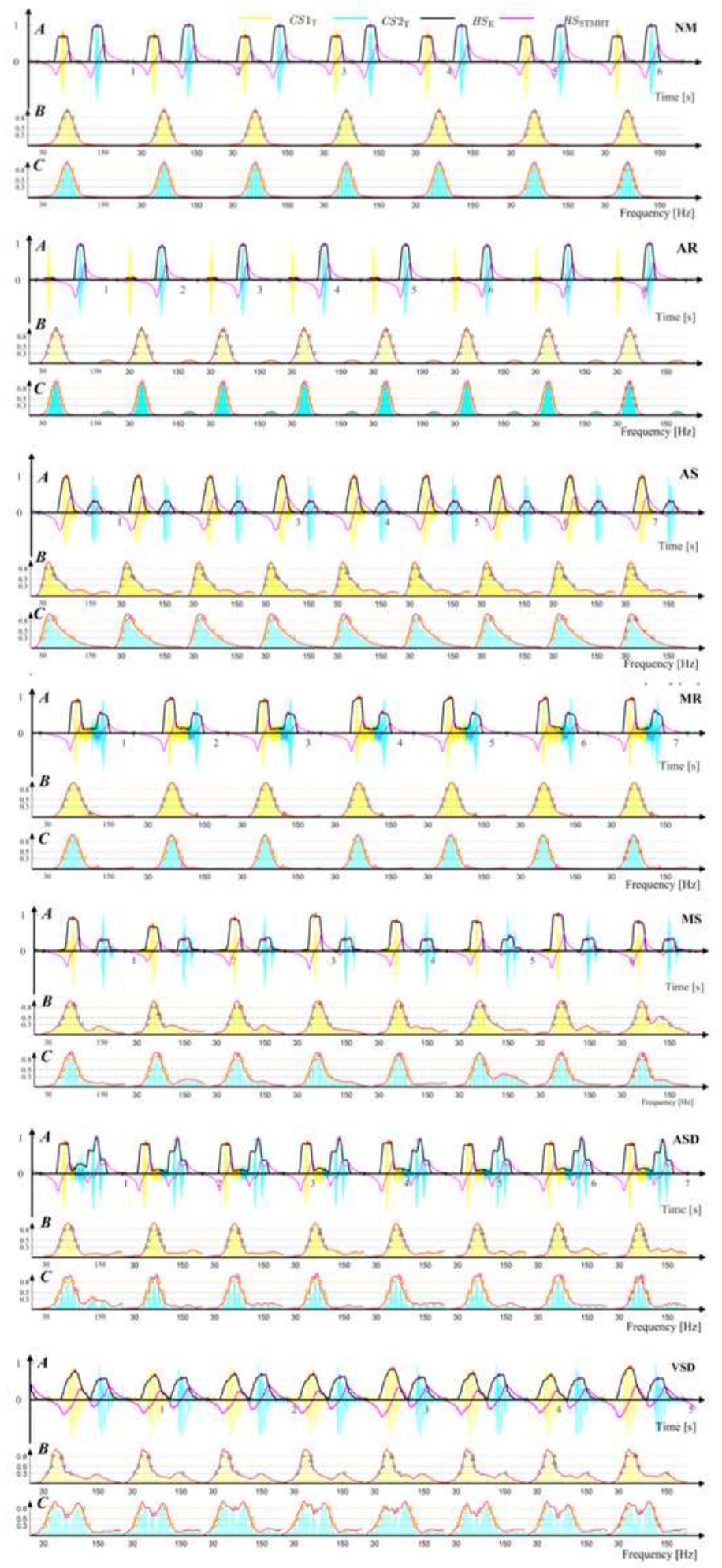

Figure 4

Examples of a typical normal sound and six types of typical heart disease sounds. 

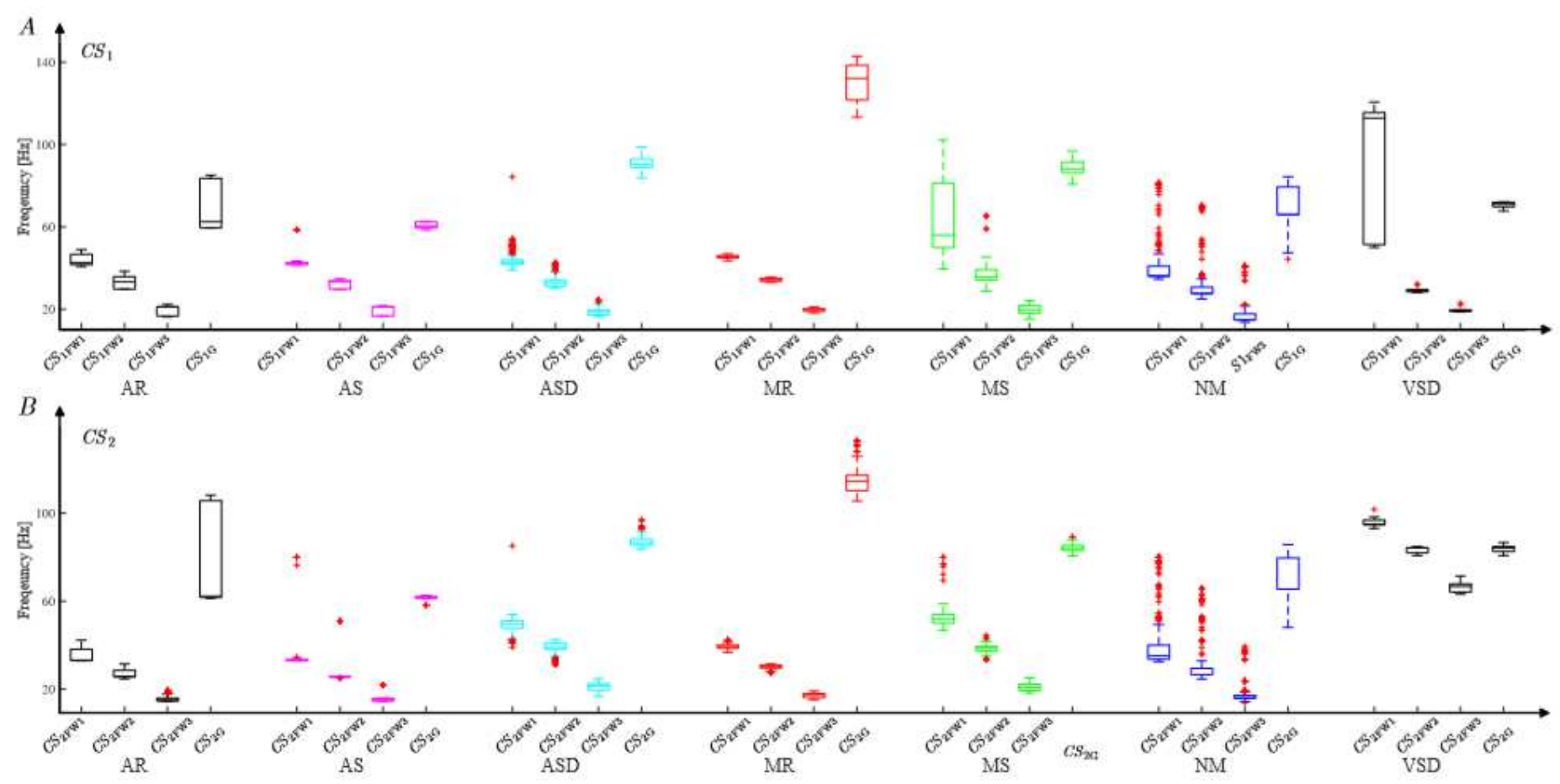

Figure 5

Box plot representation of FF for each type of heart disease. A shows the box plots for features from CS1. In addition, $B$ represents the features from CS2. 


$$
\mathrm{FF}=\left[C S_{1 \mathrm{FW}}, C S_{1 \mathrm{FW}_{2}}, C S_{1 \mathrm{FW}}, C S_{1 \mathrm{G}}, C S_{2 \mathrm{FW}_{1}}, C S_{2 \mathrm{FW}_{2}}, C S_{2 \mathrm{FW}}, C S_{2 \mathrm{G}}\right]
$$

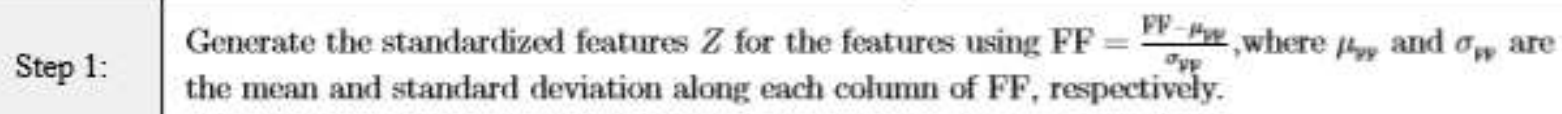

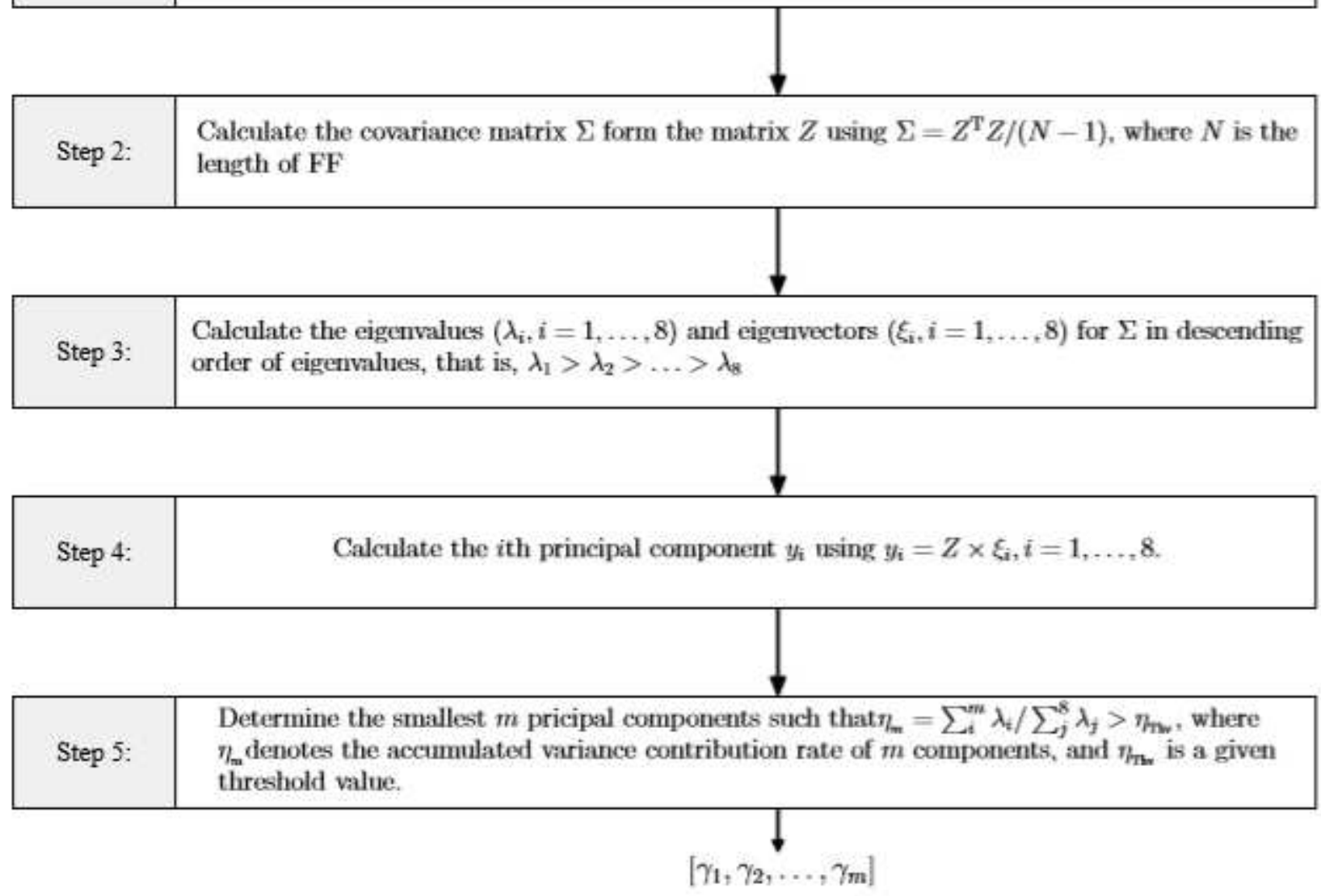

\section{Figure 6}

The flow chart corresponding to the efficient feature generation procedure. 

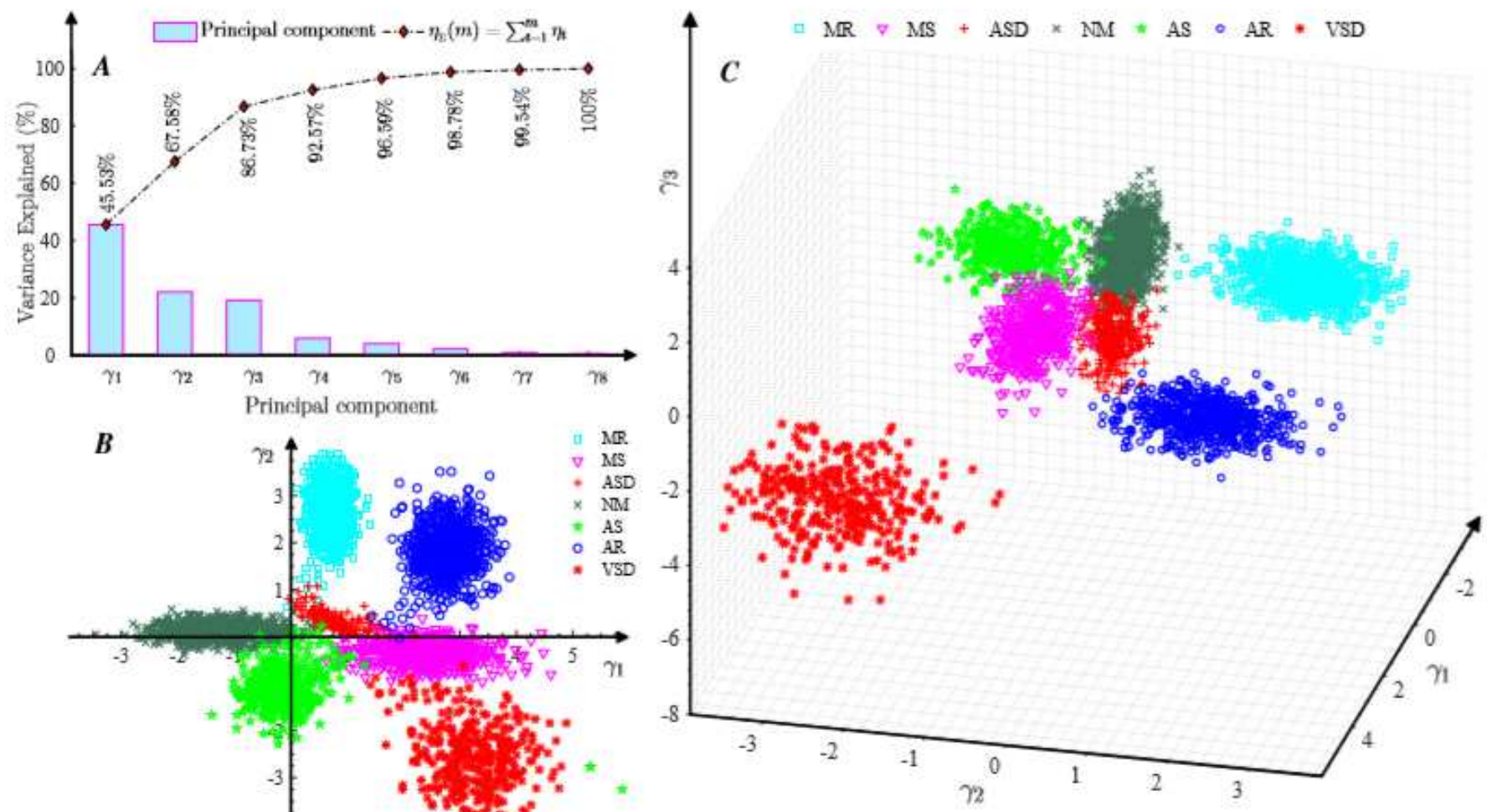

\section{Figure 7}

PCA results. A shows the Pareto chart of the variance by contribution of each principal component, $B$ plots the scatter diagram of the first two components $\gamma 1$ and $\gamma 2$, and $\mathrm{C}$ shows the scatter diagram of the first three components $\gamma 1, \gamma 2$ and $\gamma 3$.
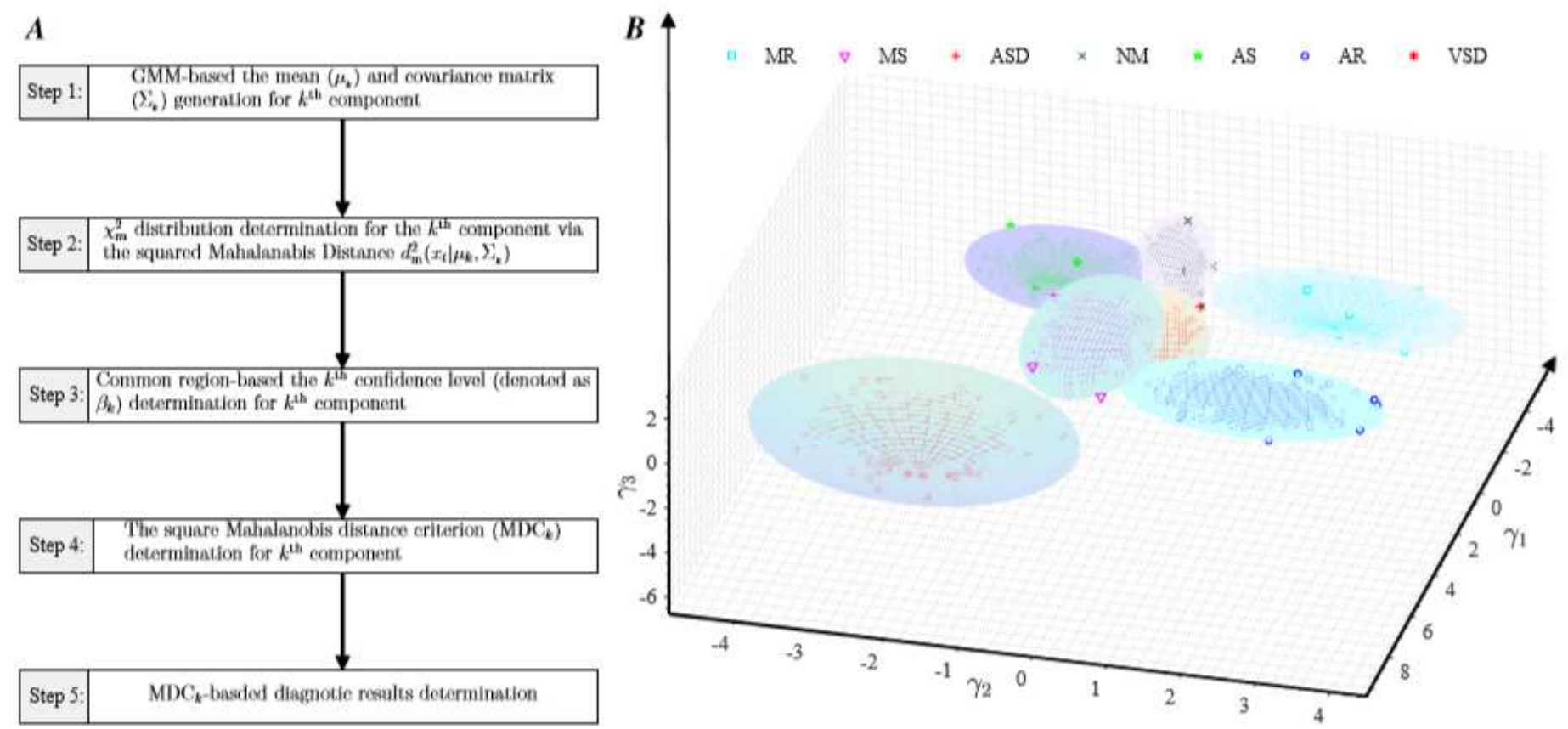
Flow chart of the diagnostic determination and 3-dimensional surface classifier results.
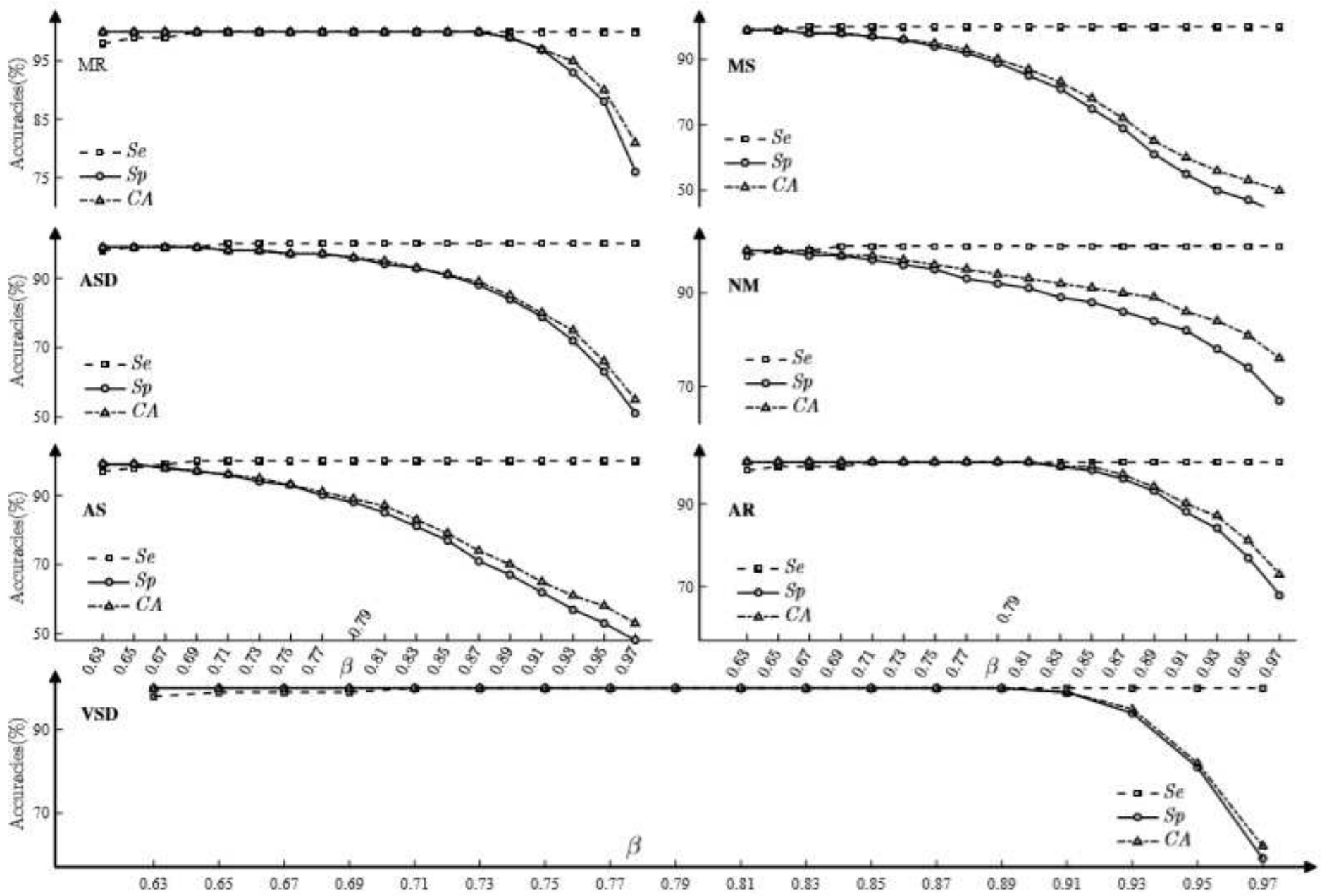

Figure 9

The achieved accuracies corresponding to classifying the heart sounds described in subsection by setting $\beta$ form 0.63 to 0.97 with a step of 0.02 . 